\title{
A Stochastic Model for Electron Transfer in Bacterial Cables
}

\author{
Nicolò Michelusi, Sahand Pirbadian, Mohamed Y. El-Naggar and Urbashi Mitra
}

\begin{abstract}
Biological systems are known to communicate by diffusing chemical signals in the surrounding medium. However, most of the recent literature has neglected the electron transfer mechanism occurring amongst living cells, and its role in cellcell communication. Each cell relies on a continuous flow of electrons from its electron donor to its electron acceptor through the electron transport chain to produce energy in the form of the molecule adenosine triphosphate, and to sustain the cell's vital operations and functions. While the importance of biological electron transfer is well-known for individual cells, the past decade has also brought about remarkable discoveries of multicellular microbial communities that transfer electrons between cells and across centimeter length scales, e.g., biofilms and multicellular bacterial cables. These experimental observations open up new frontiers in the design of electron-based communications networks in microbial communities, which may coexist with the more well-known communication strategies based on molecular diffusion, while benefiting from a much shorter communication delay. This paper develops a stochastic model that links the electron transfer mechanism to the energetic state of the cell. The model is also extensible to larger communities, by allowing for electron exchange between neighboring cells. Moreover, the parameters of the stochastic model are fit to experimental data available in the literature, and are shown to provide a good fit.
\end{abstract}

\section{INTRODUCTION}

Biological systems are known to communicate by diffusing chemical signals in the surrounding medium. One example is quorum sensing [2]-[4], where the concentration of certain signature chemical compounds emitted by the bacteria is used to estimate the bacterial population size, so as to simultaneously activate a certain collective behavior. More recently, molecular communication has been proposed as a viable communication scheme for nanodevices and nanonetworks, and is under IEEE standards consideration [5]. The performance evaluation, optimization and design of molecular communications systems opens up new challenges in the information theory [6]-[9]. The achievable capacity of the chemical channel using molecular communication is investigated in [10], [11], under Brownian motion, and in [12], under a diffusion channel. In [13], a new architecture for networks of bacteria to form a data collecting

N. Michelusi and U. Mitra are with the Ming Hsieh Department of Electrical Engineering, University of Southern California, Los Angeles, USA M. Y. El-Naggar and S. Pirbadian are with the Department of Physics and Astronomy, University of Southern California, Los Angeles, USA; emails: \{michelus, spirbadi, mnaggar, ubli\}@usc.edu

N. Michelusi and U. Mitra acknowledge support from one or all of these grants: ONR N00014-09-1-0700, CCF-0917343, CCF-1117896, CNS 1213128, AFOSR FA9550-12-1-0215, and DOT CA-26-7084-00. S. Pirbadian and M. Y. El-Naggar acknowledge support from NASA Cooperative Agreement NNA13AA92A and grant DE-FG02-13ER16415 from the Division of Chemical Sciences, Geosciences, and Biosciences, Office of Basic Energy Sciences of the US Department of Energy. N. Michelusi is in part supported by AEIT (Italian association of electrical engineering) through the research scholarship "Isabella Sassi Bonadonna 2013".

Parts of this work have appeared in [1]. network is described, and aspects such as reliability and speed of convergence of consensus are investigated. In [14], [15], a new molecular modulation scheme for nanonetworks is proposed and analyzed, based on the idea of time-sharing between different types of molecules in order to effectively suppress the interference. In [16], an in-vitro molecular communication system is designed and, in [17], an energy model is proposed, based on molecular diffusion.

While communication via chemical signals has been the focus of most prior investigations, experimental evidence on the microbial emission and response to three physical signals, i.e., sound waves, electromagnetic radiation and electric currents, suggests that physical modes of microbial communication could be widespread in nature [18]. In particular, communication exploiting electron transfer in a bacterial network has previously been observed in nature [19] and in bacterial colonies in lab [20]. This multi-cellular communication is usually triggered by extreme environmental conditions, e.g., lack of electron donor (ED) or electron acceptor (EA), in turn resulting in various gene expression levels and functions in different cells within the community, and enables the entire community to survive under harsh conditions. Electron transfer is fundamental to cellular respiration: each cell relies on a continuous flow of electrons from an ED to an EA through the cell's electron transport chain (ETC) to produce energy in the form of the molecule adenosine triphosphate (ATP), and to sustain its vital operations and functions. This strategy, known as oxidative phosphorylation, is employed by all respiratory microorganisms. In this regard, we can view the flow of one electron from the ED to the EA as an energy unit which is harvested from the surrounding medium to power the operations of the cell, and stored in an internal "rechargeable battery" (energy queue, e.g., see the literature on energy harvesting for wireless communications and references therein [21]-[23]).

While the importance of biological electron transfer and oxidative phosphorylation is well-known for individual cells, the past decade has also brought about remarkable discoveries of multi-cellular microbial communities that transfer electrons between cells and across much larger length scales than previously thought [24]. Within the span of only a few years, observations of microbial electron transfer have jumped from nanometer to centimeter length scales, and the structural basis of this remarkably long-range transfer has evolved from recently discovered molecular assemblies known as bacterial nanowires [24]-[26], to entire macroscopic architectures, including biofilms and multi-cellular bacterial cables, consisting of thousands of cells lined up end-to-end in marine sediments [19], [27] (see Fig. 11). Therein, the cells in the deeper regions of the sediment where the ED is located extract more electrons, while the cells in the upper layers, where Oxygen (an EA) is 


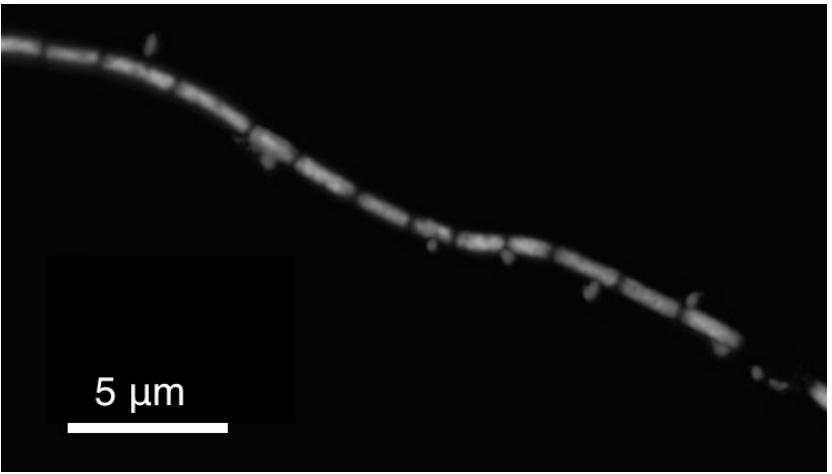

Figure 1. Fluorescent image of filamentous Desulfobulbaceae. Bacterial cells are aligned to form the cable, which couples Oxygen reduction at the marine sediment surface to sulphide reduction in deeper anoxic layers by transferring electrons along its length. From [19].

more abundant, have a heightened transfer of electrons to the EA. The survival of the whole system relies on this division of labor, with the intermediate cells operating as "relays" of electrons to coordinate this collective response to the spatial separation of ED and EA. It is worth noticing that other biological cable-like mechanisms exist in nature, enabling cellcell communication: tunneling nanotubes connect two animal cells for transport of organelles and membrane vesicles and create complex networks of interconnected cells [28]; in the bacterial world, Myxococcus xanthus cells form membrane tubes that connect cells to one another in order to transfer outer membrane content [29].

These experimental observations raise the possibility of an electron-based communications network in microbial communities, which may coexist with the more well-known communication strategies based on molecular diffusion [14], [18], [24]. For microbes, the advantage of electron-based communications is clear: in contrast to the relatively slow diffusion of whole molecules via Brownian motion, electron transfer is a rapid process that enables cells to quickly sense and respond to their environment. As an example of a communications architecture based on electron transfer, consider a system composed of an ED terminal (transmitter, or electron source) which operates as the signal encoder, an EA terminal (receiver, or electron sink) and the network of bacteria; the electron signal, encoded by the ED terminal and input into the network, is then relayed in a multi-hop fashion, following the natural laws of electron transfer within each cell and across neighboring cells, which this paper aims at modeling; the flow of electrons is finally collected at the EA terminal. Such an electron signal, coupled with the energetic state of each cell, can be "decoded" by the individual cells to activate a certain desired gene expression. For instance, in a biofilm formed on a surface, bacteria interact with each other and with a solid phase terminal EA via electron transfer, which serves both as a respiratory advantage and a communications scheme for bacteria to adapt to their environment. Additionally, electron transfer can be employed in place of molecular diffusion for quickly transporting information in nanonetworks. In particular, information can be encoded in the concentration of electrons released by the encoder into the bacterial cable, using a technique termed concentration shift keying [14], [30]. The additional challenge with respect to molecular diffusion is that the electron is both an energy carrier involved in the energy production for the cell to sustain its functionalities, and an information carrier, which enables the transport of information between nanodevices, thus introducing additional constraints in the encoded signal.

Electron-based communication presents significant advantages, as discussed above, but this phenomenon also raises new intriguing questions. While a single cell can extract enough free energy to power life's reactions by exploiting the redox potential difference between ED oxidation and EA reduction, how can the same potential difference be used to power an entire multicellular assembly such as the Desulfobulbaceae bacterial cables [19]? Specifically, can intermediate cells survive without access to chemical ED or EA, by exploiting the potential difference between cells in the deeper sediment (sulfide oxidizers) and cells in the oxic zone (oxygen reducers)? For a cable consisting of thousands of cells this appears unlikely, since the free energy available for an intermediate cell is inversely proportional to the total number of cells. Are additional, yet unknown, electron sources and sinks necessary to maintain the whole community? These questions necessitate flexible models that analyze emerging experimental data in order to elucidate the energetics of individual cells, as presented here, and, eventually, whole bacterial cables or biofilms.

In order to enable the modeling and control of such microbial communications network and guide future experiments, in this paper we set out to develop a stochastic queuing theoretic model that links electron transfer to the energetic state of the cell (e.g., ATP concentration or energy charge potential). We show how the proposed model can be extended to larger communities (e.g., cables, biofilms), by allowing for electron transfer between neighboring cells. In particular, we analyze the stochastic model for an isolated cell, which is the building block of multi-cellular networks, and provide an example of the application of the proposed framework to the computation of the cell's lifetime. Finally, we design a parameter estimation framework and fit the parameters of the model to experimental data available in the literature. The prediction curves are compared to experimental ones, showing a good fit. This paper represents a preliminary essential modeling step towards the design and analysis of bacterial communications networks, and provides the ground to model and control bacterial interactions (e.g., gene expressions) induced by the electron transfer signal, and to analyze information theoretic aspects, such as the interplay between information capacity and lifetime of the cells, as well as communication reliability and delay.

This paper is organized as follows. In Sec. II we present a stochastic model for the cell, and for the interconnection of cells via electron transfer. In Sec. III, we specialize the model to the case of an isolated cell. In Sec. IV] we present an application of the proposed framework to compute the lifetime of an isolated cell. In Sec. V, we present a parameter estimation framework and fit the parameters of the model to experimental data. Finally, Sec. VI presents some future work and Sec. VII concludes the paper. 


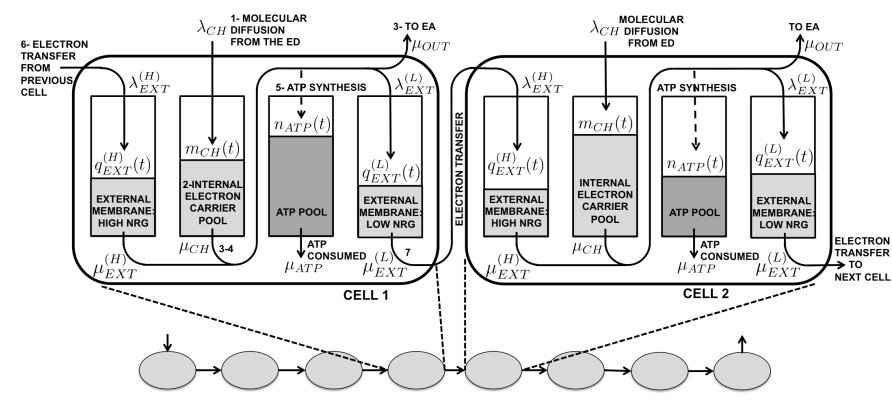

Figure 2. Stochastic model of electron transfer within a bacterial cable.

\section{Stochastic CELL MODEL}

In this section, we describe a continuous time stochastic model for the dynamics of electron flow and ATP production and consumption within a single cell, represented in Fig. 2 . This is the building block of more complex multi-cellular systems, e.g., a bacterial cable, also represented in Fig. 2 The cell is modeled as a system with an input electron flow coming either from the ED via molecular diffusion, or from a neighboring cell via electron transfer, and an output flow of electrons leaving either toward the EA via molecular diffusion, or toward the next cell in the cable via electron transfer. We first review these well known biological and physical mechanisms and then provide our new stochastic model. Inside the cell, the conventional pathway of electron flow, enabled by the presence of the ED and the EA, is as follows (see the numbers in Fig. 2):

1) ED molecules permeate inside the cell via molecular diffusion;

2) The presence of these ED molecules inside the cell results in reactions that produce electron-containing carriers (e.g., NADH). These are collected in the internal electron carrier pool (IECP, Fig. 2). The electron carriers diffusively transfer electrons to the ETC, which is partially localized in the cell inner membrane;

3) The electrons originating from the electron carriers flow through the ETC and are discarded by either a soluble and internalized EA (e.g., molecular Oxygen) or are transferred through the periplasm to the outer membrane and deposited on an extracellular EA;

4) The electron flow through the ETC results in the production of a proton concentration gradient (proton motive force [31]) across the inner membrane of the cell;

5) The proton motive force is utilized by an inner membrane protein called ATP synthase to produce ATP as an energy reserve that will later be used for various functions in the cell. The ATP produced in this way is collected in the ATP pool in Fig. 2, and used by the cell to sustain its vital operations and functions.

Alternatively, when the cells are organized in multi-cellular structures, e.g., bacterial cables, an additional pathway of electron flow may exist, termed intercellular electron transfer (IET), which involves only a transfer of electrons between neighboring cells, as opposed to molecules (ED and EA) diffusing through the cell membrane. In this regime, one or both of the ED and the EA are replaced by neighboring cells in a network of interconnected cells. In other words, IET can be substituted for the ED or the EA, enabling cells to survive even in the absence of the ED or the EA. In this case, the pathway for the electrons is as follows:

6) High-energy 1 electrons localized in the outer-membrane of a neighboring cell are transported to the host cell, and utilized in its ETC to produce ATP. Therefore, the electrons creating the proton motive force are not originating from the chemical carriers such as NADH, but instead are entering directly from the neighboring cell;

7) The electrons subsequently leave the ETC and move to the outer-membrane of the host cell, and are transferred to another neighboring cell that, in turn, uses these electrons to produce ATP.

As a result, this cooperative strategy creates a multi-cellular ETC that utilizes IET to distribute electrons throughout an entire bacterial network. These electrons originate from the ED localized on one end of the network to the locally available EA on the other end. The collective electron transport through this network provides energy for all cells involved to maintain their vital operations. The conventional ED-EA and IET processes may coexist, depending on the availability of both ED and EA in the medium where the cell is operating and on the connectivity of the cell to neighboring ones. For instance, if the concentration of ED and EA is sufficiently large, only the conventional pathway is used by the cell for ATP production. In contrast, if such concentration is too small to support ATP production, only IET from/to neighboring cells may be active. In accordance with the steps outlined above, we propose the following stochastic model for the cell, as depicted in Fig. 2 This model incorporates four pools:

1) The IECP, containing the electron carrier molecules (e.g., NADH) produced as a result of ED diffusion across the cell membrane and chemical processes occurring inside the cell;

2) The ATP pool, containing all the ATP molecules produced as a result of electron flow from the electron carriers through the ETC to the EA;

3) The external membrane pool, which involves the extracellular respiratory pathway of the cell in the outer membrane. This part of the ETC typically includes hemecontaining c-type cytochromes that facilitate electron transfer outside of the inner membrane and into the terminal EA. In fact, the accumulation of these c-type cytochromes in the outer membrane forms the external membrane pool. In order to incorporate the case of IET into this model, we assume that the external membrane pool is further divided into two parts:

a) High energy external membrane (HEEM), which contains high energy electrons coming from previous cells in the cable;

b) Low energy external membrane (LEEM), which collects low energy electrons that have been used

\footnotetext{
${ }^{1}$ Note that the terms high and low referred to the energy of electrons are used here only in relative terms, i.e., relative to the redox potential at the cell surface. In bacterial cables, the redox potential slowly decreases along the cable, thus inducing a net flow of electrons from one end to the opposite one.
} 
to synthesize ATP, before they are transferred to a neighboring cell.

Each pool in this model has a corresponding inflow and outflow of electrons that connect that pool to the others, and one cell to the next in the cable:

1) The IECP gains electrons from ED molecules diffusing into the cell and transforming into electron carriers through a series of reactions; we model this as a flow with rate $\lambda_{C H}$ joining the IECP in Fig. 2 The electrons leave this pool to the ETC (cell inner membrane) to produce ATP, modeled as another flow with rate $\mu_{C H}$ leaving the IECP in Fig. 2,

2) Alternatively, electrons are transferred from neighboring cells into the HEEM, corresponding to the flow with rate $\lambda_{E X T}^{(H)}$ in Fig. 2. These electrons leave this pool to the ETC (cell inner membrane) to produce ATP, modeled as another flow with rate $\mu_{E X T}^{(H)}$ leaving the IECP in Fig. 2 ,

3) The electron flow out of the first pool (either the IECP or the HEEM) directly causes the synthesis of ATP, so that the overall flow into the ATP pool is $\mu_{C H}+\mu_{E X T}^{(H)}$. On the other hand, ATP consumption via ATP hydrolysis within the cell through various functions is responsible for the ATP molecules leaving the ATP pool, with rate $\mu_{A T P}$

4) As a simplification, we assume that there are two major pathways for the electron output of the ETC: internalized molecular Oxygen in aerobic conditions and transport to the external membrane in anaerobic conditions. The former case, modeled as a flow with rate $\mu_{O U T}$ leaving the cell to the EA in Fig. 2, does not involve the external membrane pool but only the EA. In contrast, the latter involves the extracellular respiration pathway, which includes the external membrane. The electrons in this case are collected in the LEEM, i.e., the flow with rate $\lambda_{E X T}^{(L)}$ in Fig. 2. The electrons in this pool can, in turn, be transferred to neighboring cells, modeled as a flow with rate $\mu_{E X T}^{(L)}$ leaving the LEEM of cell 1 to the HEEM of cell 2 in Fig. 2, or to solid phase terminal EAs, not represented in Fig. 2

In addition, because typical values for transfer rates between electron carriers (e.g., outer-membrane cytochromes) on the cell exterior are relatively high [26], one can assume that the external membranes of neighboring cells have high transfer rates between one another, i.e., when IET is active, we have $\mu_{E X T}^{(L)}=\lambda_{E X T}^{(H)}=\infty$, so that any electron collected in the LEEM is instantaneously transferred to the HEEM of the neighboring cell in the cable. Under these assumptions, we can simplify the model by combining the LEEM and HEEM pools of Fig. 2 together, so that any pair of neighboring cells share a single pool for IET. On the other hand, if the cell is isolated, no IET occurs, hence $\mu_{E X T}^{(L)}=0$ and/or $\lambda_{E X T}^{(H)}=0$. This latter case will be studied in more detail in Sec. III

We model the cell as a finite state machine, and characterize the state of the cell and its stochastic evolution. The internal state of a given cell at time $t$ is defined as

$$
\mathbf{s}_{I}(t)=\left(m_{C H}(t), n_{A T P}(t), q_{E X T}^{(L)}(t), q_{E X T}^{(H)}(t)\right),
$$

where:

- $m_{C H}(t)$ is the number of electrons in the IECP that will participate in the synthesis of ATP; these electrons are carried by ED units which diffuse through the membrane into the cell (e.g., lactate), and are bonded to electron carriers within the cell (e.g., NADH); $m_{C H}(t)$ takes value in the set $\mathcal{M}_{C H} \equiv\left\{0,1, \ldots, M_{C H}\right\}$, where $M_{C H}$ is the electro-chemical storage capacity of the cell;

- $n_{A T P}(t)$ is the number of ATP molecules within the cell, taking value in the set $\mathcal{N}_{A X P} \equiv\left\{0,1, \ldots, N_{A X P}\right\}$, where $N_{A X P}$ is the overall number of ATP plus ADP molecules in the cell, which is assumed to be constant over time; $N_{A X P}$ also represents the maximum number of ATP molecules which can be present within the cell at any time (when no ADP is present);

- $q_{E X T}^{(H)}(t)$ is the number of electrons in the HEEM, taking value in the set $\mathcal{Q}_{E X T}^{(H)} \equiv\left\{0,1, \ldots, Q_{E X T}^{(H)}\right\}$, where $Q_{E X T}^{(H)}$ is the electron "storage capacity" of the HEEM;

- $q_{E X T}(t)$ is the number of electrons in the LEEM, taking value in the set $\mathcal{Q}_{E X T}^{(L)} \equiv\left\{0,1, \ldots, Q_{E X T}^{(L)}\right\}$, where $Q_{E X T}^{(L)}$ is the electron "storage capacity" of the LEEM.

Remark 1 For simplicity, we assume that all the quantities related to the state of the cell and to the flows of electrons/molecules are in terms of equivalent number of electrons involved, rather than molecular units. Hence, for instance, the ATP level in the ATP pool, $n_{A T P}(t)$, actually represents the equivalent number of electrons involved in the synthesis of the corresponding quantity of ATP available in the cell. Similarly, the level of $N A D H$ in the IECP, $m_{C H}(t)$, is expressed in terms of the equivalent number of electrons carried by the electron carriers, which actively synthesize ATP. A similar interpretation holds for the flows (of electrons, rather than molecules or $\mathrm{mM}$, where M stands for "1 molar"). Transition from one representation (electrons) to the other (molecules or $\mathrm{mM}$ ) is possible by appropriate scaling.

Moreover, while in the following analysis we assume that one "unit" corresponds to one electron, this can be generalized to the case where one "unit" corresponds to $N_{E}$ electrons, so that, e.g., $n_{A T P}$ units in the ATP pool correspond to $N_{E} n_{A T P}$ electrons.

Note that, if the cell is connected to other cells in a larger community, the low (respectively, high) energy external membrane is shared with the high (low) energy external membrane of the neighboring cell, owing to the high transfer rate approximation, as explained above. Additionally, we denote the state of death of the cell as DEAD (to be specified later). The state space of the cell is denoted as

$$
\mathcal{S}_{I} \equiv\left(\mathcal{M}_{C H} \times \mathcal{N}_{A X P} \times \mathcal{Q}_{E X T}^{(L)} \times \mathcal{Q}_{E X T}^{(H)}\right) \cup\{\mathrm{DEAD}\} .
$$

Note that the behavior of the cell is influenced by the concentration of the ED and the EA in the surrounding medium. Therefore, we also define the external state of the cell as $\mathbf{s}_{E}(t)=\left(\sigma_{D}(t), \sigma_{A}(t)\right)$, where $\sigma_{D}(t)$ and $\sigma_{A}(t)$ are, respectively, the external concentration of the ED and the EA. For simplicity, we assume that $\mathbf{s}_{E}(t)$ is an exogenous process, 


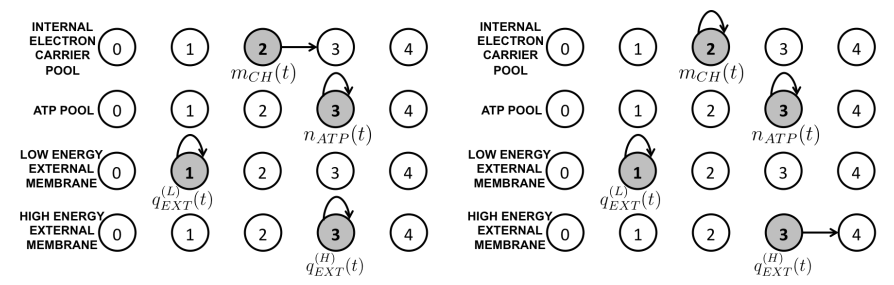

(a) ED diffusion: One electron is (b) IET: One electron is collected in transported by the ED through the the HEEM via IET from a neighboring cell membrane and is collected in the cell

IECP

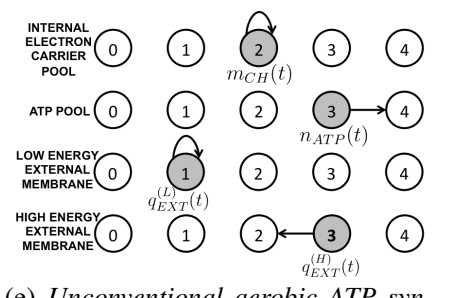

(e) Unconventional aerobic ATP synthesis: One electron is taken from the
HEEM to synthesize ATP, and is then HEEM to synthesize
captured by an EA

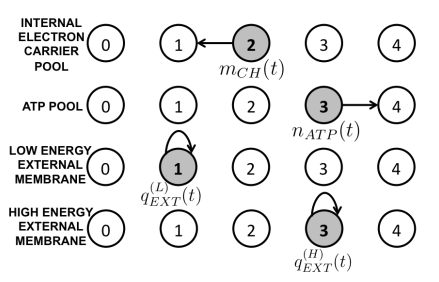

(c) Conventional aerobic ATP synthesis: One electron is taken from the the captured by an EA, leaving the cell

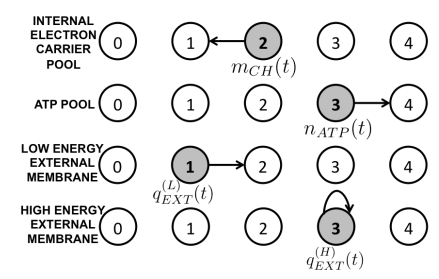

(d) Conventional anaerobic ATP synthesis: One electron is taken from the IECP to synthesize ATP, and is then collected in the LEEM

Figure 3. Markov chain and transitions from state $\mathbf{s}_{I}(t)=(2,3,1,3)$, i.e., two electrons are in the IECP, three ATP units are in the ATP pool, one electron is in the LEEM, and three electrons are in the HEEM, respectively (see Fig. 2)

not influenced by the cell dynamics, i.e., the consumption of the ED and the EA by the cell does not influence their concentration in the surrounding medium. This requires that the medium in which cells are suspended is continuously being replaced by fresh medium containing a constant amount of the ED and the EA. Otherwise a high cell concentration would use up all the resources in the time-scales relevant to this model. This aspect will be considered in future work, and is beyond the scope of the current paper.

The internal state process of cell $i, \mathbf{s}_{I}^{(i)}(t) \in \mathcal{S}_{I}$ (see Eq. 1), is time-varying and stochastic; $\mathbf{s}_{I}^{(i)}(t)$ evolves as a consequence of electro/chemical reactions occurring within the cell, chemical diffusion through the cell membrane, and IET from the neighboring cell $i-1$ to the neighboring cell $i+1$. The evolution of $\mathbf{s}_{I}^{(i)}(t)$ is also influenced by the external state $\mathbf{s}_{E}^{(i)}(t)$ experienced by the cell. We define the following processes affecting the evolution of $\mathbf{s}_{I}^{(i)}(t)$, all of which, for analytical tractability, are modeled as Poisson processes with state-dependent rates; these processes are represented in Fig. 2 and the corresponding state transitions are depicted in Fig. 3 .

- ED diffusion through the membrane: ED molecules carry electrons to synthesize ATP, which are stored in the IECP; this process occurs with rate $\lambda_{C H}\left(\mathbf{s}_{I}^{(i)}(t) ; \mathbf{s}_{E}^{(i)}(t)\right)$ [electrons/s]. Whenever an ED diffuses through the membrane within the cell (say, at time $t$ ), the state $m_{C H}^{(i)}(t)$ increases by one unit (Fig. 3 a), so that the internal state moves from $\mathbf{s}_{I}^{(i)}(t)=\left(m_{C H}, n_{A T P}, q_{E X T}^{(L)}, q_{E X T}^{(H)}\right)$ at time $t$ to $\mathbf{s}_{I}^{(i)}\left(t^{+}\right)=\left(m_{C H}+1, n_{A T P}, q_{E X T}, q_{E X T}\right)$ at time instant $t^{+}$;

- IET from the neighboring cell $i-1$ : the electron is collected in the HEEM, so that the corresponding state increases by one unit and $\mathbf{s}_{I}^{(i)}\left(t^{+}\right)=$ $\left(m_{C H}, n_{A T P}, q_{E X T}^{(L)}, q_{E X T}^{(H)}+1\right)$ (Fig. 3. b); note that this process is coupled with the anaerobic ATP synthesis (see definition below) process of the neighboring cell $i-1$ from which the electron is transferred; in fact, owing to the high transfer rate approximation, the LEEM of cell $i-1$ is shared with the HEEM of cell $i$, so that the rate of electron flow into the HEEM of cell $i$ is $\lambda_{E X T}^{(L)}\left(\mathbf{s}_{I}^{(i-1)}(t) ; \mathbf{s}_{E}^{(i-1)}(t)\right)$;

- Conventional ATP synthesis: this process involves the transfer of one electron from the IECP to the internal membrane to synthesize ATP, with rate $\mu_{C H}\left(\mathbf{s}_{I}^{(i)}(t), \mathbf{s}_{E}^{(i)}(t)\right)$ [electrons/s]. Correspondingly, one molecule of ATP is generated; the electron then leaves the internal membrane and follows either the aerobic pathway (i.e., it is captured by an internalized EA, such as Oxygen, see Fig. 3. c), with overall rate $\mu_{O U T}\left(\mathbf{s}_{I}^{(i)}(t) ; \mathbf{s}_{E}^{(i)}(t)\right)$, or the anaerobic one (Fig. 3 d) and is collected in the LEEM, with overall rate $\lambda_{E X T}\left(\mathbf{s}_{I}^{(i)}(t) ; \mathbf{s}_{E}^{(i)}(t)\right.$ ) (note that this is also the HEEM of cell $i+1)$. If the aerobic pathway is followed, the new state becomes $\mathbf{s}_{I}^{(i)}\left(t^{+}\right)=$ $\left(m_{C H}-1, n_{A T P}+1, q_{E X T}^{(L)}, q_{E X T}^{(H)}\right)$ (Fig. 3. c). Otherwise (anaerobic pathway), the new state becomes $\mathbf{s}_{I}^{(i)}\left(t^{+}\right)=$ $\left(m_{C H}-1, n_{A T P}+1, q_{E X T}^{(L)}+1, q_{E X T}^{(H)}\right)$ (Fig. 3 d);

- Unconventional ATP synthesis: this process involves the transfer of one electron from the HEEM to the internal membrane to synthesize ATP, with rate $\mu_{E X T}^{(H)}\left(\mathbf{s}_{I}^{(i)}(t), \mathbf{s}_{E}^{(i)}(t)\right) \quad$ [electrons/s]. Afterwards, the electron follows a similar path as in the conventional ATP synthesis, i.e., either it is captured by an internalized EA (aerobic pathway), with overall rate $\mu_{O U T}\left(\mathbf{s}_{I}^{(i)}(t) ; \mathbf{s}_{E}^{(i)}(t)\right)$, or it is collected in the LEEM of the cell (anaerobic pathway), with overall rate $\lambda_{E X T}^{(L)}\left(\mathbf{s}_{I}^{(i)}(t) ; \mathbf{s}_{E}^{(i)}(t)\right)$. In the former case, the new state becomes $\mathbf{s}_{I}^{(i)}\left(t^{+}\right)=\left(m_{C H}, n_{A T P}+1, q_{E X T}^{(L)}, q_{E X T}^{(H)}-1\right)$ 
(Fig. 3e); in the latter, $\mathbf{s}_{I}^{(i)}\left(t^{+}\right)=\left(m_{C H}, n_{A T P}+1, q_{E X T}^{(L)}+1, q_{E X T}^{(H)} \quad-\quad 1\right)$ (Fig. 3. f);

- ATP consumption: this process provides energy for cellular functions, and occurs with rate $\mu_{A T P}\left(\mathbf{s}_{I}^{(i)}(t) ; \mathbf{s}_{E}^{(i)}(t)\right)$ [electrons/s]; when one molecule of ATP is consumed, the state $n_{A T P}^{(i)}(t)$ decreases by one unit, so that $\mathbf{s}_{I}^{(i)}\left(t^{+}\right)=$ $\left(m_{C H}, n_{A T P}-1, q_{E X T}^{(L)}, q_{E X T}^{(H)}\right)$ (Fig. 3 g);

- $\underline{\text { Death }}$ process, with rate $\delta\left(\mathbf{s}_{I}^{(i)}(t) ; \mathbf{s}_{E}^{(i)}(t)\right)$ : if death occurs, the new state becomes $\mathbf{s}_{I}^{(i)}\left(t^{+}\right)=$DEAD, from which the cell cannot recover any longer, i.e., $\mathbf{s}_{I}^{(i)}(\tau)=$ DEAD, $\forall \tau>t$.

\section{A. Flow Constraints}

Note that the rates of the different flows involved need to satisfy some constraints, induced by the queuing model employed. In particular, if some queue is empty (respectively, saturated), the rate of the corresponding outbound (respectively, inbound) flow must be zero, so that, for instance, for the flows out of and into the ATP pool, the following condition must hold:

$\mu_{A T P}\left(m_{C H}, 0, q_{E X T}^{(L)}, q_{E X T}^{(H)} ; \mathbf{s}_{E}\right)=0$, (outbound flow),

$\mu_{C H}\left(m_{C H}, N_{A X P}, q_{E X T}^{(L)}, q_{E X T}^{(H)} ; \mathbf{s}_{E}\right)$

$+\mu_{E X T}^{(H)}\left(m_{C H}, N_{A X P}, q_{E X T}^{(L)}, q_{E X T}^{(H)} ; \mathbf{s}_{E}\right)=0$, (inbound flow).

A similar consideration holds for the other queues and the corresponding flows. Moreover,

$\mu_{E X T}^{(H)}\left(\mathbf{s}_{I} ; \mathbf{s}_{E}\right)+\mu_{C H}\left(\mathbf{s}_{I} ; \mathbf{s}_{E}\right)=\lambda_{E X T}^{(L)}\left(\mathbf{s}_{I} ; \mathbf{s}_{E}\right)+\mu_{O U T}\left(\mathbf{s}_{I} ; \mathbf{s}_{E}\right)$,

since each electron leaving either the IECP or the HEEM to synthesize ATP either follows the aerobic pathway to the EA or the anaerobic one to the LEEM.

We further assume that

$$
\begin{aligned}
& \lambda_{C H}\left(\mathbf{s}_{I} ; \sigma_{D}, \sigma_{A}\right)=\sigma_{D} \lambda_{C H}\left(\mathbf{s}_{I} ; 1, \sigma_{A}\right), \\
& \mu_{O U T}\left(\mathbf{s}_{I} ; \sigma_{D}, \sigma_{A}\right)=\sigma_{A} \mu_{O U T}\left(\mathbf{s}_{I} ; \sigma_{D}, 1\right),
\end{aligned}
$$

thus capturing the fact that the molecular diffusion rate is proportional to the ED (respectively, EA) concentration. This assumption is supported by Fick's law of diffusion [32], which states that the diffusion rate is linearly dependent on the concentration differential between inside and outside. It follows that, if no ED is present $\left(\sigma_{D}=0\right)$, then $\lambda_{C H}\left(\mathbf{s}_{I} ; 0, \sigma_{A}\right)=0$ and no ED diffusion may occur. Similarly, if no EA is present $\left(\sigma_{A}=0\right)$, then $\mu_{O U T}\left(\mathbf{s}_{I} ; \sigma_{D}, 0\right)=0$ and no EA diffusion may occur. In Sec. V-A, a parametric model for these flows is presented, based on which the model is fit to experimental data.

\section{ISOLATED CELL MODEL}

In the most general case, electron transport in a series of interconnected single-cell organisms is represented by the proposed stochastic model. However, this model can also explain the electron transport behavior of a single cell, which
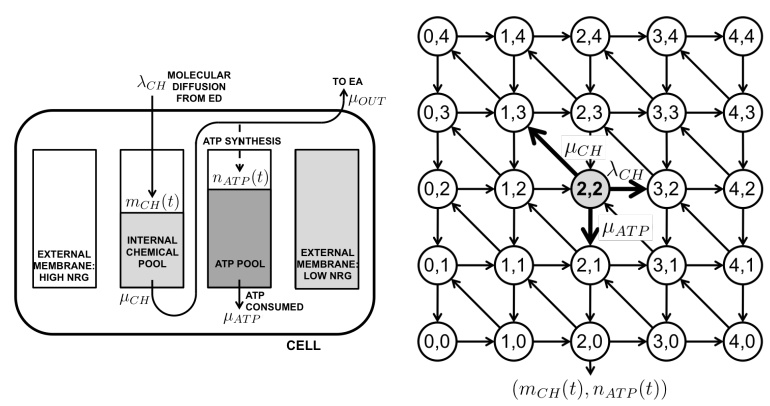

Figure 4. Stochastic model for an isolated cell, after the transient phase during which the HEEM gets depleted and the LEEM gets charged (left), and Markov chain with the corresponding transitions (right), for the case where $M_{C H}=4, N_{A X P}=4$. The transition rates from state $(2,2)$ are also depicted.

is the building block of the general multi-cell system. The experimental investigation of a multi-cellular network of bacteria is very challenging, in fact:

1) In order to build a chain of interconnected cells, singlecell organisms have to be placed in each other's proximity. Placing multiple cells next or close to each other in a controlled way that maintains the intercellular contact is very difficult in practice and requires cellular manipulation techniques such as optical tweezers [33], as well as nanofabricated micron-scaled chambers designed specifically to hold these communities in place;

2) In vivo characterization of the energetic and electron transfer properties of an individual cell within this chain independently from the other cells requires complex chemical and optical assays that have never been used in such complicated systems.

Therefore, instead of the most general case of the model (multi-cell system), we start by investigating the properties of single, isolated cells. Using a few simplifying assumptions, the general model can be reduced to a single cell model which can be more easily matched against experimental results. In addition, the single-cell experiments are not hindered by the practical issues mentioned above, which makes them easier to perform. In this way, we can characterize the properties of the individual components, which will help us better understand the electron transport in multi-cellular systems.

In the case of an isolated cell, the IET process is not active, and $\lambda_{E X T}^{(H)}(t)=\mu_{E X T}^{(L)}(t)=0$. As a result, the HEEM gets depleted, and the LEEM gets filled. Therefore, after a transient phase, the cell reaches the configuration depicted in Fig. 4. where the HEEM is empty, and the LEEM is fully charged. In the following treatment, we assume that the transient phase is concluded, hence $q_{E X T}^{(L)}(t)=Q_{E X T}^{(L)}$ and $q_{E X T}^{(H)}(t)=0, \forall t$, so that the state $\left(q_{E X T}^{(L)}(t), q_{E X T}^{(H)}(t)\right)=\left(Q_{E X T}^{(L)}, 0\right)$ of the external membrane can be neglected. Assuming that the cell operates in this configuration, we thus redefine its internal state as $\mathbf{s}_{I}(t)=\left(m_{C H}(t), n_{A T P}(t)\right)$.

The corresponding Markov chain and state transitions are depicted in Fig. 4. From the continuous-time process described in Sec. II, we now generate a discrete-time process, as detailed below. Initially, we assume that the external state $\mathbf{s}_{E}(t)$ is fixed, i.e., $\sigma_{D}(t)=\sigma_{D}, \forall t$ and $\sigma_{A}(t)=\sigma_{A}, \forall t$. The case 
where $\mathbf{s}_{E}(t)$ is piecewise constant will be considered in Sec. III-D. The discretization is obtained by sampling the state process $\mathbf{s}_{I}(t)$ at specific times, corresponding to one of the events described in Sec. II. specialized to the case of an isolated cell: molecular diffusion; conventional aerobic ATP synthesis; ATP consumption; death. Starting from time $t=0$ in state $\mathbf{s}_{I}(0) \in \mathcal{S}_{I}$, we define $T_{k}$ as the time instant corresponding to the occurrence of the $k$ th event since time 0 , and $\mathbf{S}_{k}$ as the corresponding state at time instant $T_{k}^{+}$(i.e., right after the corresponding transition occurs). In particular, $T_{0}=0$ and $\mathbf{S}_{0}=\mathbf{S}_{I}(0)$. Note that, by sampling, we have transformed the continuous-time stochastic process into a discrete-time Markov chain, with finite state space $\mathcal{S}_{I}$. However, the duration of the $k$ th time-slot, $T_{k+1}-T_{k}$, is not fixed but is a random variable which depends on the inter-arrival time of the events described in Sec. III-D In the subsequent sections, we first derive the transition probabilities of the underlying discrete time Markov chain and the inter-arrival times of the events, thus leading to a full-characterization of the stochastic dynamics of $\mathbf{s}_{I}(t)$. We then provide an example of applicability of this framework to the computation of the lifetime of the cell. Finally, in Sec. V we present a parameter estimation framework and match the model to experimental data available in [34].

\section{A. Transition Probabilities and inter-arrival times}

In this section, we compute the transition probability of the underlying discrete-time Markov chain, and the distribution of the inter-arrival times in the corresponding continuous time system. To this end, let $\mathbf{S}_{k}=\sqrt{2} \in \mathcal{S}_{I} \backslash\{$ DEAD $\}$ be the state of the cell at time $T_{k}^{+}$. We compute the transition probability

$$
\mathbb{P}\left(\mathbf{S}_{k+1}=j, T_{k+1}>\tau \mid \mathbf{S}_{k}=i, T_{k}=t\right),
$$

for some $j \in \mathcal{S}_{I}, \tau \geq t$ (note that, due to the memoryless property of Poisson processes, the event $\mathbf{S}_{k+1}=j, T_{k+1}>\tau$ conditioned on $\mathbf{S}_{k}=i, T_{k}=t$ is independent of the realization of $\left.\left\{\left(\mathbf{S}_{j}, T_{j}\right), 0 \leq j<k\right\}\right)$. Let $\lambda_{i, j}$ be the transition rate from state $i$ to state $j$, which depends on the specific event which triggers the transition. For instance, if $i$ corresponds to $\left(m_{C H}, n_{A T P}\right)$ and $j$ to $\left(m_{C H}, n_{A T P}-1\right)$, then a transition from state $i$ to state $j$ occurs if the ATP consumption event occurs, with rate $\lambda_{i, j}=\mu_{A T P}\left(\mathbf{s}_{I} ; \mathbf{s}_{E}\right)$. The transition from state $i$ to state $j$ can be interpreted as follows. Let $E_{i, s}$ be the event which triggers the transition from $i$ to $s$, and $t+W_{i, s}$ be the time when such event occurs (with respect to the reference time-position $t$ ). From the properties of Poisson processes, we have that $W_{i, s}$ is an exponential random variable, with pdf $f_{W_{i, s}}(w)=\lambda_{i, s} e^{-\lambda_{i, s} w}$, and that $\left\{W_{i, s}, \forall s\right\}$ are mutually independent. Then, the system moves to state $j$ if $t+W_{i, j}<t+W_{i, s}, \forall s \neq j$, i.e., the event $E_{i, j}$ is the first one to occur, which thus triggers the transition. Therefore, the probability (4) is equivalent to

$$
\begin{aligned}
& \mathbb{P}\left(\mathbf{S}_{k+1}=j, T_{k+1}>\tau \mid \mathbf{S}_{k}=i, T_{k}=t\right) \\
& =\mathbb{P}\left(t+W_{i, j}>\tau, W_{i, j}<W_{i, s}, \forall s \neq j \mid \mathbf{S}_{k}=i, T_{k}=t\right) \\
& =\int_{\tau-t}^{\infty} \lambda_{i, j} e^{-\lambda_{i, j} w} \prod_{s \neq j} \mathbb{P}\left(W_{i, s}>w\right) \mathrm{d} w=\frac{\lambda_{i, j}}{R_{i}} e^{-R_{i}(\tau-t)},
\end{aligned}
$$

\footnotetext{
${ }^{2}$ In this section, $i$ is an index corresponding to a specific state in $\mathcal{S}_{I}$.
}

where we have defined the total flow from state $i, R_{i}=$ $\sum_{s} \lambda_{i, s}$, we have marginalized with respect to $W_{i, j}$, we have used the independence among $\left\{W_{i, s}, \forall s\right\}$ and $\mathbb{P}\left(W_{i, s}>w\right)=$ $e^{-\lambda_{i, s} w}$. From [5, we thus obtain the transition probability $\mathbb{P}\left(\mathbf{S}_{k+1}=j \mid \mathbf{S}_{k}=i\right)$ by letting $\tau=t$ in 5 and by noticing that the resulting expression is independent of $t$, i.e.,

$\mathbb{P}\left(\mathbf{S}_{k+1}=j \mid \mathbf{S}_{k}=i, T_{k}=t\right)=\frac{\lambda_{i, j}}{R_{i}}=\mathbb{P}\left(\mathbf{S}_{k+1}=j \mid \mathbf{S}_{k}=i\right)$.

We now compute the distribution of the inter-arrival time $T_{k+1}-T_{k}$ as

$$
\begin{aligned}
& \mathbb{P}\left(T_{k+1}-T_{k}>\tau-t \mid \mathbf{S}_{k}=i, \mathbf{S}_{k+1}=j, T_{k}=t\right) \\
& =\frac{\mathbb{P}\left(\mathbf{S}_{k+1}=j, T_{k+1}>\tau \mid \mathbf{S}_{k}=i, T_{k}=t\right)}{\mathbb{P}\left(\mathbf{S}_{k+1}=j \mid \mathbf{S}_{k}=i, T_{k}=t\right)}=e^{-R_{i}(\tau-t)}
\end{aligned}
$$

Note that the resulting expression is independent of $\mathbf{S}_{k+1}$ and of time $t$, since the process is stationary. We can thus write

$$
\mathbb{P}\left(T_{k+1}-T_{k}>\tau-t \mid \mathbf{S}_{k}=i\right)=e^{-R_{i}(\tau-t)} .
$$

We define the $\left(\left|\mathcal{S}_{I}\right|-1\right) \times\left(\left|\mathcal{S}_{I}\right|-1\right)$ transition probability matrix $\mathbf{T}$ of the underlying discrete-time Markov chain within $\mathcal{S}_{I} \backslash\{$ DEAD $\}$, with entries $\mathbf{T}(i, j)=\mathbb{P}\left(\mathbf{S}_{k+1}=j \mid \mathbf{S}_{k}=\right.$ $i), i, j \in \mathcal{S}_{I} \backslash\{\mathrm{DEAD}\}$ (we do not consider transitions from DEAD, since this is absorbing). The transition probability from $i \in \mathcal{S}_{I} \backslash\{\mathrm{DEAD}\}$ to DEAD is then given by $1-\mathbf{e}_{i}^{T} \mathbf{T} \mathbf{1}$, where $\mathbf{1}$ is the column vector of all ones, and $\mathbf{e}_{i}$ equals 1 in the position corresponding to state $i$, and zero otherwise.

\section{B. State distribution of the system at time $t>0$}

Given the analysis of the underlying discrete-time Markov chain and of the inter-arrival times in the previous section, we are now able to compute the state distribution of the system at a generic time $t$, given that $\mathbf{S}_{I}(0)=i$. We define

$$
\mathbb{P}_{t}(j \mid i)=\mathbb{P}\left(\mathbf{S}_{I}(t)=j \mid \mathbf{S}_{I}(0)=i\right), j \in \mathcal{S}_{I} \backslash\{\mathrm{DEAD}\} .
$$

In order to compute it, let $0<h<t$. By the memoryless property of Poisson processes,

$$
\begin{aligned}
\mathbb{P}_{t}(j \mid i) & =\sum_{s \in \mathcal{S}_{I} \backslash\{\mathrm{DEAD}\}} \mathbb{P}\left(\mathbf{S}_{I}(t)=j, \mathbf{S}_{I}(t-h)=s \mid \mathbf{S}_{I}(0)=i\right) \\
& =\sum_{s \in \mathcal{S}_{I} \backslash\{\mathrm{DEAD}\}} \mathbb{P}_{h}(j \mid s) \mathbb{P}_{t-h}(s \mid i) .
\end{aligned}
$$

It follows that, $\forall i, j \in \mathcal{S}_{I} \backslash\{\mathrm{DEAD}\}$,

$$
\mathbb{P}_{t}(j \mid i)-\mathbb{P}_{t-h}(j \mid i)=\sum_{s \in \mathcal{S}_{I} \backslash\{\mathrm{DEAD}\}}\left(\mathbb{P}_{h}(j \mid s)-\delta_{j, s}\right) \mathbb{P}_{t-h}(s \mid i)
$$

Then, dividing by $h$ and taking the limit for $h \rightarrow 0$, we obtain

$$
\frac{\mathrm{d} \mathbb{P}_{t}(j \mid i)}{\mathrm{d} t}=\sum_{s \in \mathcal{S}_{I} \backslash\{\mathrm{DEAD}\}} \lim _{h \rightarrow 0} \frac{\mathbb{P}_{h}(j \mid s)-\delta_{j, s}}{h} \mathbb{P}_{t}(s \mid i) .
$$

Note that $\lim _{h \rightarrow 0} \frac{\mathbb{P}_{h}(j \mid s)-\delta_{j, s}}{h}=\lambda_{s, j}$, and $\lim _{h \rightarrow 0} \frac{\mathbb{P}_{h}(s \mid s)-\delta_{s, s}}{h}=-R_{s}$. Substituting in (9), we obtain the system of differential equations

$$
\frac{\mathrm{d} \mathbb{P}_{t}(j \mid i)}{\mathrm{d} t}=\sum_{s \in \mathcal{S}_{I} \backslash\{\mathrm{DEAD}, j\}} \lambda_{s, j} \mathbb{P}_{t}(s \mid i)-R_{j} \mathbb{P}_{t}(j \mid i), \quad \forall i, j
$$


Letting $\mathbf{P}_{t}$ be the $\left(\left|\mathcal{S}_{I}\right|-1\right) \times\left(\left|\mathcal{S}_{I}\right|-1\right)$ matrix with components $\mathbf{P}_{t}(i, j)=\mathbb{P}_{t}(j \mid i), i, j \in \mathcal{S}_{I} \backslash\{\mathrm{DEAD}\}$, we can rewrite the system of differential equations (10) as

$$
\mathbf{P}_{t}^{\prime}=\mathbf{P}_{t} \mathbf{A},
$$

where we have defined the flow matrix $\mathbf{A}$ with components $\mathbf{A}(s, j)=\lambda_{s, j}$ for $j \neq s$ and $\mathbf{A}(j, j)=-R_{j}$, and $\mathbf{P}_{t}^{\prime}$ represents the first-order derivative of $\mathbf{P}_{t}$ with respect to time. Note that $\mathbf{A}=\mathbf{R}(\mathbf{T}-\mathbf{I})$, where $\mathbf{T}$ is the transition matrix of the underlying discrete-time Markov chain within $\mathcal{S}_{I} \backslash\{\mathrm{DEAD}\}$, derived in the previous section, $\mathbf{R}$ is the rate matrix, a diagonal matrix with entries $\mathbf{R}(i, i)=R_{i}$, and $\mathbf{I}$ is the unit matrix. Moreover, by Gershgorin's circle Theorem [35], all eigenvalues of $\mathbf{A}$ are non-positive. The general solution to 11 subject to $\mathbf{P}_{0}=\mathbf{I}$ is

$$
\mathbf{P}_{t}=\exp \{\mathbf{A} t\}
$$

where we have defined the matrix exponential $\exp \{\mathbf{A} t\}=$ $\sum_{k=0}^{\infty} \frac{t^{k}}{k !} \mathbf{A}^{k}$. Note that such solution guarantees a feasible transition probability matrix, i.e., $\left[\mathbf{P}_{t}\right]_{i, j} \geq 0$ and $\sum_{j}\left[\mathbf{P}_{t}\right]_{i, j} \leq 1$.

\section{Numerical evaluation of $\mathbf{P}_{t}$}

Unfortunately, from our numerical evaluations, we have verified that $\mathbf{A}$ can seldom be diagonalized. Therefore, we employ an alternative solution to efficiently compute $\mathbf{P}_{t}$. Let $\Delta \ll 1$ and $n=\lceil t / \Delta\rceil$. Then, the general solution can be approximated as

$\mathbf{P}_{t}=[\exp \{\mathbf{A} \Delta\}]^{n} \exp \{\mathbf{A}(t-\Delta n)\} \simeq[\exp \{\mathbf{A} \Delta\}]^{n}=\mathbf{P}_{\Delta}^{n}$,

where we have used the approximation $\exp \{\mathbf{A}(t-\Delta n)\} \simeq \mathbf{I}$, which holds for $\Delta \ll 1$. Moreover, since we assume $\Delta \ll 1$, we approximate the matrix exponential $\mathbf{P}_{\Delta}=\exp \{\mathbf{A} \Delta\}$ with the first order Taylor approximation

$$
\mathbf{P}_{\Delta} \simeq \mathbf{I}+\Delta \mathbf{A}=\mathbf{I}-\Delta \mathbf{R}(\mathbf{I}-\mathbf{T}) \triangleq \tilde{\mathbf{P}}_{\Delta} .
$$

Note that the approximation $\tilde{\mathbf{P}}_{\Delta}$ of $\mathbf{P}_{\Delta}$ is a feasible transition matrix with non-negative entries, if $\Delta<\min _{i}\left\{1 / R_{i}\right\}$.

\section{Extension to $\mathbf{s}_{E}(t)$ piecewise constant}

In this section, we extend the previous analysis to the case where the external ambient state is piecewise constant, i.e., $\mathbf{s}_{E}(t)=\mathbf{s}_{E, n}, \forall n \in\left[\tau_{n}, \tau_{n+1}\right), \forall n \geq 0$, where $0=\tau_{0}$ and $\tau_{n}<\tau_{n+1}, \forall n \geq 0$. This analysis is of interest for the following experimental evaluation: the ED concentration is varied in order to measure the response in terms of fluctuations in the ATP level within the cell.

For this case, it is straightforward to derive the probability of the cell being in state $\mathbf{s}_{I}(t)=j \in \mathcal{S}_{I} \backslash\{$ DEAD $\}$ at time $t \in$ $\left[\tau_{n}, \tau_{n+1}\right)$, for some $n \geq 0$, given $\mathbf{s}_{I}(0)=i \in \mathcal{S}_{I} \backslash\{\mathrm{DEAD}\}$. To this end, let $\mathbf{T}_{n}$ be the transition probability matrix within $\mathcal{S}_{I} \backslash\{\mathrm{DEAD}\}, \mathbf{R}_{n}$ be the rate matrix, and $\mathbf{A}_{n}=\mathbf{R}_{n}\left(\mathbf{T}_{n}-\mathbf{I}\right)$ be the flow matrix when $\mathbf{s}_{E}(t)=\mathbf{s}_{E, n}$. Then, $\forall t \in\left[\tau_{n}, \tau_{n+1}\right)$ we have

$$
\mathbf{P}_{t}=\left[\prod_{m=0}^{n-1} \exp \left\{\mathbf{A}_{m}\left(\tau_{m+1}-\tau_{m}\right)\right\}\right] \times \exp \left\{\mathbf{A}_{n}\left(t-\tau_{n}\right)\right\} .
$$

where we have defined $\prod_{m=0}^{n-1} \mathbf{C}_{m}=\mathbf{C}_{0} \times \mathbf{C}_{1} \times \cdots \times \mathbf{C}_{n-1}$, and we have used the fact that, from the Markov property,

$$
\begin{aligned}
& \mathbb{P}\left(\mathbf{s}_{I}(t)=j \mid \mathbf{s}_{I}(0)=i\right)=\sum_{s_{0}, s_{1}, \ldots, s_{n} \in \mathcal{S}_{I} \backslash\{\mathrm{DEAD}\}} \mathbb{P}\left(\mathbf{s}_{I}(t)=j \mid \mathbf{s}_{I}\left(\tau_{n}\right)=s_{n}\right) \\
& \times \prod_{m=0}^{n-1} \mathbb{P}\left(\mathbf{s}_{I}\left(\tau_{m+1}\right)=s_{m+1} \mid \mathbf{s}_{I}\left(\tau_{m}\right)=s_{m}\right),
\end{aligned}
$$

and, since $\mathbf{s}_{E}(\tau)$ is constant in the time interval $\left[\tau_{m}, \tau_{m+1}\right)$, the probability $\mathbb{P}\left(\mathbf{s}_{I}\left(\tau_{m+1}\right)=s_{m+1} \mid \mathbf{s}_{I}\left(\tau_{m}\right)=s_{m}\right)$ can be computed as in Sec. III-A

\section{APPLiCATION TO CELL-LifETIME COMPUTATION, ISOLATED CELL}

For every cell in the bacterial chain, it is possible that, at some point in time, due to variations in the energetic state of the cell and changes to the supply of the ED and the EA, the cell reaches a state where its ATP consumption rate reaches a minimum value (e.g., zero). Once a cell enters this state, it is considered dead and its ATP consumption rate may not restore to normal values, thus jeopardizing the overall functionality of the cable. Accordingly, the time it takes for a cell to reach this irreversible state is defined as the lifetime of the cell. This quantity can be measured experimentally by using indicators of cellular respiratory activity. In an experimental setup where cells in a bacterial chain can be characterized on an individual basis, cellular lifetime is one of the easiest measurable quantities that contains a significant amount of information regarding the specific properties of the target cell. In this section, we apply the stochastic model presented in Sec. III to the computation of the lifetime of an isolated cell, defined as follows.

\section{Definition 1 The lifetime of the cell, L, is defined as}

$$
L=\min \left\{t>0: \mathbf{S}_{I}(t)=\operatorname{DEAD}\right\} .
$$

Equivalently, letting $k^{*}=\min \left\{k>0: \mathbf{S}_{k}=\mathrm{DEAD}\right\}$, we have $L=T_{k^{*}}$.

In this section, we compute the probability density function (pdf) of the lifetime, $f_{L}\left(t ; \boldsymbol{\pi}_{0}\right)$, as well as the expected lifetime $\mathbb{E}\left[L \mid \boldsymbol{\pi}_{0}\right]$, given some initial state distribution $\boldsymbol{\pi}_{0}(i), i \in \mathcal{S}_{I} \backslash$ $\{$ DEAD $\} . f_{L}\left(t ; \pi_{0}\right)$ is given by (we use $\mathbb{P}$ to denote also a pdf)

$$
\begin{aligned}
& f_{L}\left(t ; \boldsymbol{\pi}_{0}\right)=\mathbb{P}\left(L=t \mid \boldsymbol{\pi}_{0}\right) \\
& =\sum_{k=0}^{\infty} \mathbb{P}\left(L=t, \text { Death occurs at the }(k+1) \text { th event } \mid \boldsymbol{\pi}_{0}\right) .
\end{aligned}
$$

Note that the event $(L=t$,Death occurs at the $(k+1)$ th event) is equivalent to

$$
\mathbf{S}_{k} \in \mathcal{S}_{I} \backslash\{\mathrm{DEAD}\}, \mathbf{S}_{k+1}=\mathrm{DEAD}, T_{k+1}=t,
$$

i.e., the cell is alive upon occurrence of the $k$ th event, and dies upon occurrence of the $(k+1)$ th event. Therefore, we obtain

$$
f_{L}\left(t ; \boldsymbol{\pi}_{0}\right)=\sum_{k=0}^{\infty} \sum_{i \in \mathcal{S}_{I} \backslash\{\mathrm{DEAD}\}} g_{k}(i, t),
$$


where we have defined $g_{k}(i, t) \triangleq \mathbb{P}\left(\mathbf{S}_{k}=i, \mathbf{S}_{k+1}=\right.$ DEAD, $\left.T_{k+1}=t \mid \boldsymbol{\pi}_{0}\right)$. In order to compute $g_{k}(i, t)$, we first determine, for $k \geq 0$ and $s \in \mathcal{S}_{I} \backslash\{\mathrm{DEAD}\}$,

$$
h_{k}(s, t) \triangleq \mathbb{P}\left(\mathbf{S}_{k}=s, T_{k}=t \mid \boldsymbol{\pi}_{0}\right) .
$$

For $k=0$, this is given by $h_{0}(s, t)=\pi_{0}(s) \delta(t)$, where $\delta(t)$ is the Kronecker delta function. For $k>0$, we have

$$
\begin{aligned}
& h_{k}(s, t)=\sum_{j} \int_{0}^{t} \mathbb{P}\left(\mathbf{S}_{k}=s, T_{k}=t, \mathbf{S}_{k-1}=j, T_{k-1}=\tau \mid \boldsymbol{\pi}_{0}\right) \mathrm{d} \tau \\
& =\sum_{j} \int_{0}^{t} \mathbb{P}\left(T_{k}-T_{k-1}=t-\tau \mid \mathbf{S}_{k-1}=j\right) \\
& \quad \times \mathbb{P}\left(\mathbf{S}_{k}=s \mid \mathbf{S}_{k-1}=j, T_{k-1}=\tau\right) h_{k-1}(j, \tau) \mathrm{d} \tau \\
& =\sum_{j} \int_{0}^{t} R_{j} e^{-R_{j}(t-\tau)} \mathbf{T}(j, s) h_{k-1}(j, \tau) \mathrm{d} \tau
\end{aligned}
$$

It follows that

$$
\begin{aligned}
& g_{k}(s, t)=\mathbb{P}\left(\mathbf{S}_{k}=s, \mathbf{S}_{k+1}=\mathrm{DEAD}, T_{k+1}=t\right) \\
& =\int_{0}^{t} \mathbb{P}\left(\mathbf{S}_{k}=s, \mathbf{S}_{k+1}=\mathrm{DEAD}, T_{k+1}=t, T_{k}=\tau\right) \mathrm{d} \tau \\
& =\int_{0}^{t} R_{s} e^{-R_{s}(t-\tau)}\left(1-\sum_{j} \mathbf{T}(s, j)\right) h_{k}(s, \tau) \mathrm{d} \tau,
\end{aligned}
$$

where $1-\sum_{j} \mathbf{T}(s, j)$ is the transition probability to state DEAD, from state $s$. Substituting in (18), we obtain

$f_{L}\left(t ; \boldsymbol{\pi}_{0}\right)=\sum_{s} \int_{0}^{t} R_{s} e^{-R_{s}(t-\tau)}\left(1-\sum_{j} \mathbf{T}(s, j)\right) H(s, \tau) \mathrm{d} \tau$

where we have defined

$$
\begin{aligned}
& H(s, t) \triangleq \sum_{k=0}^{\infty} f_{k}(s, t) \\
& =h_{0}(s, t)+\sum_{j} \int_{0}^{t} R_{j} e^{-R_{j}(t-\tau)} \mathbf{T}(j, s) H(j, \tau) \mathrm{d} \tau \\
& =\boldsymbol{\pi}_{0}(s) \delta(t)+\sum_{j} \int_{0}^{t} R_{j} e^{-R_{j}(t-\tau)} \mathbf{T}(j, s) H(j, \tau) \mathrm{d} \tau .
\end{aligned}
$$

Then, we obtain

$$
\begin{aligned}
& \mathbb{E}[L]=\int_{0}^{\infty} t \sum_{s} \int_{0}^{t} R_{s} e^{-R_{s}(t-\tau)}\left(1-\sum_{j} \mathbf{T}(s, j)\right) H(s, \tau) \mathrm{d} \tau \mathrm{d} t \\
& =\sum_{s} \int_{0}^{\infty} R_{s} e^{R_{s} \tau}\left(1-\sum_{j} \mathbf{T}(s, j)\right) H(s, \tau) \int_{\tau}^{\infty} t e^{-R_{s} t} \mathrm{~d} t \mathrm{~d} \tau .
\end{aligned}
$$

Using the fact that $\int_{\tau}^{\infty} t e^{-R_{s} t} \mathrm{~d} t=\frac{e^{-R_{s} \tau}}{R_{s}}\left(\tau+\frac{1}{R_{s}}\right)$, we obtain

$$
\begin{aligned}
& \mathbb{E}[L]=\sum_{s}\left(1-\sum_{j} \mathbf{T}(s, j)\right) \int_{0}^{\infty} \tau H(s, \tau) \mathrm{d} \tau \\
& \quad+\frac{1}{R_{s}} \sum_{s}\left(1-\sum_{j} \mathbf{T}(s, j)\right) \int_{0}^{\infty} H(s, \tau) \mathrm{d} \tau \\
& =\sum_{s}\left(1-\sum_{j} \mathbf{T}(s, j)\right) Q(s) \\
& \quad+\sum_{s} \frac{1-\sum_{j} \mathbf{T}(s, j)}{R_{s}} \sum_{k=0}^{\infty} \mathbb{P}\left(S_{k}=s \mid \boldsymbol{\pi}_{0}\right),
\end{aligned}
$$

where we have defined $Q(s) \triangleq \int_{0}^{\infty} \tau H(s, \tau) \mathrm{d} \tau$. This term can be computed as

$$
\begin{aligned}
& Q(s)=\int_{0}^{\infty} t H(s, t) \mathrm{d} t \\
& =\sum_{j} R_{j} \mathbf{T}(j, s) \int_{0}^{\infty} e^{R_{j} \tau} H(j, \tau) \int_{\tau}^{\infty} t e^{-R_{j} t} \mathrm{~d} t \mathrm{~d} \tau \\
& =\sum_{j} \mathbf{T}(j, s) \int_{0}^{\infty} H(j, \tau)\left(\tau+\frac{1}{R_{j}}\right) \mathrm{d} \tau \\
& =\sum_{j} \mathbf{T}(j, s) Q(j)+\sum_{j} \frac{\mathbf{T}(j, s)}{R_{j}} \sum_{k=0}^{\infty} \mathbb{P}\left(S_{k}=j \mid \boldsymbol{\pi}_{0}\right) .
\end{aligned}
$$

Let $\mathbf{Q}$ be a row vector with elements $Q(j)$, and $\mathbf{x}=(\mathbf{I}-\mathbf{T}) \mathbf{1}$ be the column vector associated to transitions from the transient states to the DEAD state. We obtain

$$
Q(s)=\mathbf{Q} \mathbf{e}_{s}=\mathbf{Q} \mathbf{T} \mathbf{e}_{s}+\boldsymbol{\pi}_{0}^{T}(\mathbf{I}-\mathbf{T})^{-1} \mathbf{R}^{-1} \mathbf{T} \mathbf{e}_{s},
$$

where we have used the fact that $\sum_{k=0}^{\infty} \mathbb{P}\left(S_{k}=s \mid \boldsymbol{\pi}_{0}\right)=$ $\boldsymbol{\pi}_{0}^{T}(\mathbf{I}-\mathbf{T})^{-1} \mathbf{e}_{s}$. Therefore, we obtain

$$
\mathbf{Q}=\boldsymbol{\pi}_{0}^{T}(\mathbf{I}-\mathbf{T})^{-1} \mathbf{R}^{-1} \mathbf{T}(\mathbf{I}-\mathbf{T})^{-1} .
$$

Substituting in the expression of the expected lifetime, we obtain

$$
\mathbb{E}[L]=\boldsymbol{\pi}_{0}^{T}(\mathbf{I}-\mathbf{T})^{-1} \mathbf{R}^{-1}(\mathbf{I}-\mathbf{T})^{-1} \mathbf{x} .
$$

Finally, we use the fact that $\mathbf{x}=(\mathbf{I}-\mathbf{T}) \mathbf{1}$, yielding the expression of the expected lifetime

$$
\mathbb{E}[L]=\boldsymbol{\pi}_{0}^{T}(\mathbf{I}-\mathbf{T})^{-1} \mathbf{R}^{-1} \mathbf{1} .
$$

\section{Parameter estimation and Experimental VALIDATION}

As an example of experiments related to our stochastic model, Ozalp et al. [34] have measured in vivo levels of ATP and NADH in the yeast Saccharomyces cerevisiae, as they abruptly add ED to a suspension of starved yeast cells. Since we have theoretically investigated the single cell system, this work, which is performed on a culture of mutuallyindependent yeast cells, can be used as a test for our stochastic model.

Although the ETC in yeast is not exactly identical to the bacterial counterpart, the principles on which the model is 
based upon are conserved between yeast and bacteria. These include the involvement of an ED, electron carriers such as NADH, ETC and an EA, which in the case of yeast is molecular Oxygen.

We have extracted the measured quantities from [34] in the form of ATP and NADH concentrations as a function of time. In accordance with [34], we have assumed that yeast cells are initially starved and, at some point in time, the ED is added to the cell suspension. This triggers an increase in ATP and $\mathrm{NADH}$ production as well as ATP consumption. In extracting the data, we have averaged out the small oscillations in NADH and ATP concentrations in time, since these are mainly caused by an enzyme involved in the metabolic pathway that is specific to yeast and does not exist in the bacterial strain that we are interested in, Shewanella oneidensis MR-1. Therefore, in matching the experimental data from yeast to our model, we have only taken into account the large scale variations of the levels of ATP and NADH over time.

Let $\left\{\left(\mathbf{s}_{I, k}^{i}, t_{k}\right), k=0,1, \ldots, N\right\}$ be the time-series of the state of cell $i$ at times $t_{k}$, where $0=t_{0}<t_{1}<\cdots<t_{N}$. Let $\mathbf{s}_{E}(t)$ be the known profile of the concentration of the external ED and EA, which we assume to be piecewise constant, as in Sec. III-D and the same for all cells. In particular, we assume that $\mathbf{s}_{E}(t)=\mathbf{s}_{E, k}, \forall t \in\left[t_{k}, t_{k+1}\right), \forall k=0,1, \ldots, N-1$, so that the external state is constant in the time interval between two consecutive measurements. The measurement collected in [34] at time $t_{k}$ is

$$
\begin{aligned}
& \mathrm{NADH}_{k}=\alpha_{N A D H} \frac{1}{M} \sum_{i=1}^{M} m_{C H}^{i}\left(t_{k}\right)+\tilde{w}_{k}^{(N A D H)}, \\
& \operatorname{ATP}_{k}=\alpha_{A T P} \frac{1}{M} \sum_{i=1}^{M} n_{A T P}^{i}\left(t_{k}\right)+\tilde{w}_{k}^{(A T P)}
\end{aligned}
$$

where $\mathrm{NADH}_{k}$ is the measurement of NADH (typically, fluorescence level [34]), whereas $\mathrm{ATP}_{k}$ is the measurement of ATP (typically, in $\mathrm{mM}[34]$ ); the constants $\alpha_{N A D H}$ and $\alpha_{A T P}$ account for the conversion in the unit of measurements of NADH and ATP, respectively, from the stochastic model presented in this paper (electron units) to the experimental setup (fluorescence level and $\mathrm{mM}$, respectively); and $\tilde{w}_{k}^{(N A D H)}$ and $\tilde{w}_{k}^{(A T P)}$ are zero mean Gaussian noise samples, each i.i.d. over time, with variance $\sigma_{N A D H}^{2}$ and $\sigma_{A T P}^{2}$, respectively. A practical assumption is that $M \gg 1$, so that $\frac{1}{M} \sum_{i=1}^{M} m_{C H}^{i}\left(t_{k}\right) \simeq \mathbb{E}\left[m_{C H}\left(t_{k}\right)\right]$ and $\frac{1}{M} \sum_{i=1}^{M} n_{A T P}^{i}\left(t_{k}\right) \simeq$ $\mathbb{E}\left[n_{A T P}\left(t_{k}\right)\right]$, where the expectation is computed with respect to the state distribution at time $t_{k}$, given by $\boldsymbol{\pi}_{0}^{T} \mathbf{P}_{t_{k}}$. Letting $\mathbf{y}_{k}=\left[\alpha_{N A D H}^{-1} \mathrm{NADH}_{k}, \alpha_{A T P}^{-1} \mathrm{ATP}_{k}\right], \mathbf{w}_{k}=$ $\left[\alpha_{N A D H}^{-1} \tilde{w}_{k}^{(N A D H)}, \alpha_{A T P}^{-1} \tilde{w}_{k}^{(A T P)}\right]$ and $\mathbf{Z} \in \mathbb{R}^{\left(\left|\mathcal{S}_{I}\right|-1\right) \times 2}$ with $j$ th row $[\mathbf{Z}]_{j,:}=\left[m_{C H}(j), n_{A T P}(j)\right]$, where $m_{C H}(j)$ and $n_{A T P}(j)$ are the NADH and ATP levels in the state corresponding to index $j$, we thus obtain

$$
\mathbf{y}_{k}=\boldsymbol{\pi}_{0}^{T} \mathbf{P}_{t_{k}} \mathbf{Z}+\mathbf{w}_{k} \simeq \boldsymbol{\pi}_{0}^{T} \prod_{j=1}^{k} \mathbf{P}_{\Delta, j-1}^{n_{j}-n_{j-1}} \mathbf{Z}+\mathbf{w}_{k},
$$

where $\prod_{j=1}^{k} \mathbf{P}_{\Delta, j-1}^{n_{j}-n_{j-1}}=\mathbf{P}_{\Delta, 0}^{n_{1}} \times \mathbf{P}_{\Delta, 1}^{n_{2}-n_{1}} \times \cdots \times \mathbf{P}_{\Delta}^{n_{k}-n_{k-1}}$, $n_{j}=\left\lceil t_{j} / \Delta\right\rceil$, with $n_{0}=0$, and $\mathbf{P}_{\Delta, j-1}$ is the transition matrix with time-step size $\Delta$, when the external state takes value $\mathbf{s}_{E, j-1}$. In the last step, we have used the approximation (13). Herein, we assume that $\mathbf{w}_{k} \sim \mathcal{N}\left(\mathbf{0}, \sigma_{w}^{2} \mathbf{I}_{2}\right)$, i.e., $\alpha_{N A D H}^{-2} \sigma_{N A D H}^{2}=\alpha_{A T P}^{-2} \sigma_{A T P}^{2}=\sigma_{w}^{2}$.

\section{A. Parametric model}

The statistics of the system, defined by the transition probability matrix $\mathbf{P}_{t}$, is determined by the rates $\lambda_{C H}, \mu_{C H}$ and $\mu_{A T P}$. In this section, we present a parametric model for these rates, based on biological constraints. Specifically, we let

$$
\begin{aligned}
& \lambda_{C H}\left(\mathbf{s}_{I}(t) ; \mathbf{s}_{E}(t)\right)=\gamma \sigma_{D}(t)+\rho\left(1-\frac{m_{C H}(t)}{M_{C H}}\right) \sigma_{D}(t), \\
& \mu_{C H}\left(\mathbf{s}_{I}(t) ; \mathbf{s}_{E}(t)\right)=\zeta\left(1-\frac{n_{A T P}(t)}{N_{A X P}}\right), \\
& \mu_{A T P}\left(\mathbf{s}_{I}(t) ; \mathbf{s}_{E}(t)\right)=\beta \sigma_{D}(t),
\end{aligned}
$$

where $\gamma, \rho, \zeta, \beta \in \mathbb{R}_{+}$are parameters, that we want to estimate, and $\mathbb{R}_{+}$is the set of non-negative reals. The NADH generation rate $\lambda_{C H}$ primarily depends on the concentration of available ED, as explained in Sec. III-A Additionally, it depends on the number of available NAD molecules in the cell, since the ED reacts with NAD to form NADH. The more NAD molecules are available, the higher the rate of the NADH-producing reaction. Moreover, the larger the ATP level, the smaller the ATP generation rate $\mu_{C H}\left(\mathbf{s}_{I}(t) ; \mathbf{s}_{E}(t)\right)$. This is true because the ATP synthase, the protein responsible for ATP production, transforms ADP into ATP. Since the sum of ATP and ADP molecules in the cell is conserved, a higher ATP level corresponds to a lower ADP level. Therefore, as there is more ATP available in the cell, there are less ADP molecules available for ATP synthase to produce additional ATP molecules, which, in turn, results in a smaller ATP production rate. Finally, the larger the ED concentration, the larger the ATP consumption rate $\mu_{A T P}\left(\mathbf{s}_{I}(t) ; \mathbf{s}_{E}(t)\right)$. This is shown to be true experimentally, for instance in [34]. The reason behind this correlation is that cellular operations that consume $\operatorname{ATP}($ e.g., ATP-ases) are directly regulated by the ED concentration. Note that $\lambda_{C H}, \mu_{C H}$ and $\mu_{A T P}$ further need to satisfy the constraints listed in Sec. II-A. We assume that all the cells are alive throughout the experiment, and set the death rate $\delta\left(\mathbf{s}_{I}(t) ; \mathbf{s}_{E}(t)\right)=0$.

We define the parameter vector $\mathbf{x}=[\gamma, \rho, \zeta, \beta]$, which is estimated via maximum likelihood (ML) in the next section. Therefore, the flow matrix A, defined in (11), is a linear function of the entries of $\mathbf{x}$. We write such a dependence as $\mathbf{A}\left(\mathbf{x}, \mathbf{s}_{E}\right)$. Similarly, from (14), we write $\mathbf{P}_{\Delta}\left(\mathbf{x}, \mathbf{s}_{E}\right)=$ $\mathbf{I}+\Delta \mathbf{A}\left(\mathbf{x}, \mathbf{s}_{E}\right)$

\section{B. Maximum Likelihood estimate of $\mathbf{x}$}

For a given time series $\left\{\left(\mathbf{y}_{k}, t_{k}\right), k=0,1, \ldots, N\right\}$, and the piecewise constant profile of the external state $\mathbf{s}_{E}(t)$, in this section we design a ML estimator of $\mathbf{x}$. Since the initial distribution $\pi_{0}$ is unknown, we also estimate it jointly with $\mathbf{x}$. Note that, since the death rate is zero, the entries of $\boldsymbol{\pi}_{0}$ need to sum to one, i.e., $\mathbf{1}^{T} \boldsymbol{\pi}_{0}=1$. Moreover, we further enforce the constraints $\boldsymbol{\pi}_{0}^{T} \mathbf{Z}=\mathbf{y}_{0}$, i.e., the expected values of the NADH and ATP pools at time $t_{0}$ equal the measurement $\mathbf{y}_{0}$. Therefore, we have the linear equality constraint $\boldsymbol{\pi}_{0}^{T}[\mathbf{Z}, \mathbf{1}]=\left[\mathbf{y}_{0}, 1\right]$, and the inequality constraint $\pi_{0} \geq 0$ (component-wise). We 
denote the constraint set as $\mathcal{P}$, so that $\pi_{0} \in \mathcal{P}$. Due to the Gaussian observation model 30 , the ML estimate of $\left(\mathbf{x}, \boldsymbol{\pi}_{0}\right)$ is given by

$$
\left(\hat{\mathbf{x}}, \hat{\boldsymbol{\pi}}_{0}\right)=\underset{\mathbf{x} \geq 0, \boldsymbol{\pi}_{0} \in \mathcal{P}}{\arg \min } f\left(\mathbf{x}, \boldsymbol{\pi}_{0}\right),
$$

where we have defined the negative log-likelihood cost function

$$
f\left(\mathbf{x}, \boldsymbol{\pi}_{0}\right) \triangleq \frac{1}{2} \sum_{k=0}^{N}\left\|\mathbf{y}_{k}-\boldsymbol{\pi}_{0}^{T} \prod_{j=1}^{k} \mathbf{P}_{\Delta}\left(\mathbf{x}, \mathbf{s}_{E, j-1}\right)^{n_{j}-n_{j-1}} \mathbf{Z}\right\|_{F}^{2} .
$$

For a fixed $\mathbf{x}$, the optimization over $\boldsymbol{\pi}_{0}$ is a quadratic programming problem, which can be solved efficiently using, e.g., interior-point methods [36], [37]. On the other hand, for fixed $\boldsymbol{\pi}_{0}$, the optimization over $\mathbf{x}$ is a non-convex optimization problem. Therefore, we resort to a gradient descent (GD) algorithm to optimize over $\mathbf{x}$, which only guarantees convergence to a local optimum. Finally, we employ an iterative method to solve (32), i.e., we optimize over $\pi_{0}$ for the current estimate of $\mathbf{x}$, then we optimize over $\mathbf{x}$ for the current estimate of $\boldsymbol{\pi}_{0}$, and so on. The derivative of $f\left(\mathbf{x}, \boldsymbol{\pi}_{0}\right)$ with respect to $\mathbf{x}_{j}$ is given by

$$
\begin{aligned}
& {\left[\nabla_{\mathbf{x}} f\left(\mathbf{x}, \boldsymbol{\pi}_{0}\right)\right]_{j}=\frac{\mathrm{d}}{\mathrm{d} \mathbf{x}_{j}} f\left(\mathbf{x}, \boldsymbol{\pi}_{0}\right)} \\
& =-\sum_{k=0}^{N} \boldsymbol{\pi}_{0}^{T} \frac{\mathrm{d}\left[\prod_{j=1}^{k} \mathbf{P}_{\Delta}\left(\mathbf{x}, \mathbf{s}_{E, j-1}\right)^{n_{j}-n_{j-1}}\right]}{\mathrm{d} \mathbf{x}_{j}} \mathbf{Z} \\
& \times\left(\mathbf{y}_{k}-\boldsymbol{\pi}_{0}^{T} \prod_{j=1}^{k} \mathbf{P}_{\Delta}\left(\mathbf{x}, \mathbf{s}_{E, j-1}\right)^{n_{j}-n_{j-1}} \mathbf{Z}\right)^{T} .
\end{aligned}
$$

We further assume that the intervals satisfy $t_{k+1}-t_{k}=T, \forall k$, so that $n_{k}=k n, \forall k$, and we enforce $n=2^{b}$, for some integer $b>0$. This can be accomplished by appropriately choosing $\Delta \ll 1$. Then, the derivative $\frac{\mathrm{d}\left[\prod_{j=1}^{k} \mathbf{P}_{\Delta}\left(\mathbf{x}, \mathbf{s}_{E, j-1}\right)^{n}\right]}{\mathrm{d} \mathbf{x}_{j}}$ can be efficiently computed recursively as

$$
\begin{aligned}
& \frac{\mathrm{d}\left[\prod_{j=1}^{k} \mathbf{P}_{\Delta}\left(\mathbf{x}, \mathbf{s}_{E, j-1}\right)^{n}\right]}{\mathrm{d} \mathbf{x}_{j}}=\frac{\mathrm{d}\left[\prod_{j=1}^{k-1} \mathbf{P}_{\Delta}\left(\mathbf{x}, \mathbf{s}_{E, j-1}\right)^{n}\right]}{\mathrm{d} \mathbf{x}_{j}} \mathbf{P}_{\Delta}\left(\mathbf{x}, \mathbf{s}_{E, k-1}\right)^{n} \\
& +\prod_{j=1}^{k-1} \mathbf{P}_{\Delta}\left(\mathbf{x}, \mathbf{s}_{E, j-1}\right)^{n} \frac{\mathrm{d} \mathbf{P}_{\Delta}\left(\mathbf{x}, \mathbf{s}_{E, k-1}\right)^{n}}{\mathrm{~d} \mathbf{x}_{j}}
\end{aligned}
$$

where the derivative $\frac{d \mathbf{P}_{\Delta}\left(\mathbf{x}, \mathbf{s}_{E}\right)^{n}}{\mathrm{~d} \mathbf{x}_{j}}$ can be efficiently computed recursively as

$$
\begin{aligned}
& \frac{\mathrm{d} \mathbf{P}_{\Delta}\left(\mathbf{x}, \mathbf{s}_{E}\right)^{2^{b}}}{\mathrm{~d} \mathbf{x}_{j}}=\frac{\mathrm{d} \mathbf{P}_{\Delta}\left(\mathbf{x}, \mathbf{s}_{E}\right)^{2^{b-1}}}{\mathrm{~d} \mathbf{x}_{j}} \mathbf{P}_{\Delta}\left(\mathbf{x}, \mathbf{s}_{E}\right)^{2^{b-1}} \\
& +\mathbf{P}_{\Delta}\left(\mathbf{x}, \mathbf{s}_{E}\right)^{2^{b-1}} \frac{\mathrm{d} \mathbf{P}_{\Delta}\left(\mathbf{x}, \mathbf{s}_{E}\right)^{2^{b-1}}}{\mathrm{~d} \mathbf{x}_{j}}
\end{aligned}
$$

Finally, let $\hat{\mathbf{x}}_{p}$ be the estimate of $\mathbf{x}$ at the $p$ th iteration of the GD algorithm. Then, the GD algorithm updates the ML estimate of $\mathbf{x}$ as

$$
\hat{\mathbf{x}}_{p+1}=\left(\hat{\mathbf{x}}_{p}-\mu_{p} \nabla_{\mathbf{x}} f\left(\hat{\mathbf{x}}_{p}, \hat{\mathbf{e}}_{p}\right)\right)^{+},
$$

where $0<\mu_{k} \ll 1$ is the (possibly, time-varying) step size and we have defined $(v)^{+}=\max \{v, 0\}$, applied to each entry, so that a non-negativity constraint is enforced (in fact, the entries of $\mathbf{x}$ need to be non-negative).

\section{Results}

We use the algorithm outlined above to fit the parameter vector $\mathbf{x}$ to the experimental data. While, in principle, the capacities of both the IECP $\left(M_{C H}\right)$ and the ATP pool $\left(N_{A X P}\right)$ need to be estimated, we found that $M_{C H}=N_{A X P}=20$ provides a good fit, and good trade-off between convergence of the estimation algorithm and fitting. Note that the ATP capacity of the cell culture is $3.6 \mathrm{mM}$ and the concentration of cells is $10^{12}$ cells/liter (see [34]). It follows that the capacity of the ATP pool of each cell is $2.16 \times 10^{9}$ molecules/cell. Since, approximately, 2.5 ATP molecules are created by the flow of 2 electrons in the ETC (see [38, Sec. 18.6]), the ATP pool may carry $1.728 \times 10^{9}$ electrons/cell. Therefore, one "unit" in the stochastic model corresponds to $N_{E}=$ $0.864 \times 10^{8}$ electrons, or $1.08 \times 10^{8}$ ATP molecules. Similarly, since each NADH molecule carries 2 electrons which actively participate in the ETC, we have that one "unit" corresponds to $0.432 \times 10^{8} \mathrm{NADH}$ molecules. The timeseries $\left\{\left(\mathrm{ATP}_{k}, \mathrm{NADH}_{k}, t_{k}\right)\right\}$ is first extracted from [34], where $\mathrm{ATP}_{k}$ is in $\mathrm{mM}$ (stars in Fig. 5.a), $\mathrm{NADH}_{k}$ is a fluorescence level $\left(\times 10^{-6}\right.$, stars in Fig. 5 a), which we assume to be linearly proportional to the NADH level. The time-series is then converted to feasible values in the stochastic model. In particular, since the ATP capacity of the cell culture is $3.6 \mathrm{mM}$, and assuming that the IECP capacity of the cell culture is $\max _{k} \mathrm{NADH}_{k}=12.985$ [fluorescence $\times 10^{-6}$ ], i.e., the maximum level reached in the $\mathrm{NADH}$ measurements, the time-series is

$$
\begin{aligned}
& y_{k}^{(N A D H)}=\frac{\mathrm{NADH}_{k} \quad\left[\text { fluorescence } \times 10^{-6}\right]}{12.985\left[\text { fluorescence } \times 10^{-6}\right]} M_{C H}, \\
& y_{k}^{(A T P)}=\frac{\mathrm{ATP}_{k}[\mathrm{mM}]}{3.6[\mathrm{mM}]} N_{A X P},
\end{aligned}
$$

so that, from 29], $\alpha_{N A D H} \simeq 0.650$ [fluorescence $\mathrm{x} 10^{-6}$ and $\alpha_{A T P}=0.18[\mathrm{mM}]$. Note that both $y_{k}^{(N A D H)}$ and $y_{k}^{(A T P)}$ are dimensionless quantities. The time-series $\left\{\left(\mathbf{y}_{k}, t_{k}\right)\right\}$, where $\mathbf{y}_{k}=\left[y_{k}^{(N A D H)}, y_{k}^{(A T P)}\right]$, is then fed into the estimation algorithm. The EA concentration (molecular Oxygen) is assumed to be constant throughout the experiment, and sufficient to sustain reduction. On the other hand, the ED concentration profile, extrapolated from [34], is zero at time $t<80 \mathrm{~s}$, when cells are starved, $30 \mathrm{mM}$ at $t=80 \mathrm{~s}$, when glucose is added to the starved cells, and constantly decreases until it becomes zero at time $t \simeq 1300 \mathrm{~s}$, when cells become starved again.

Remark 2 In the parameter estimation, the samples after $t \simeq$ $1300 \mathrm{~s}$ are discarded, since cells are starved after that time, which, in turn, results in increased cell lysis occurring in the cell suspension. The cellular material released by lysis can be used by other intact cells as ED or EA. For this reason, and since the extent of cell lysis in unpredictable in the cell culture, the concentrations of the ED and the EA cannot be accurately 


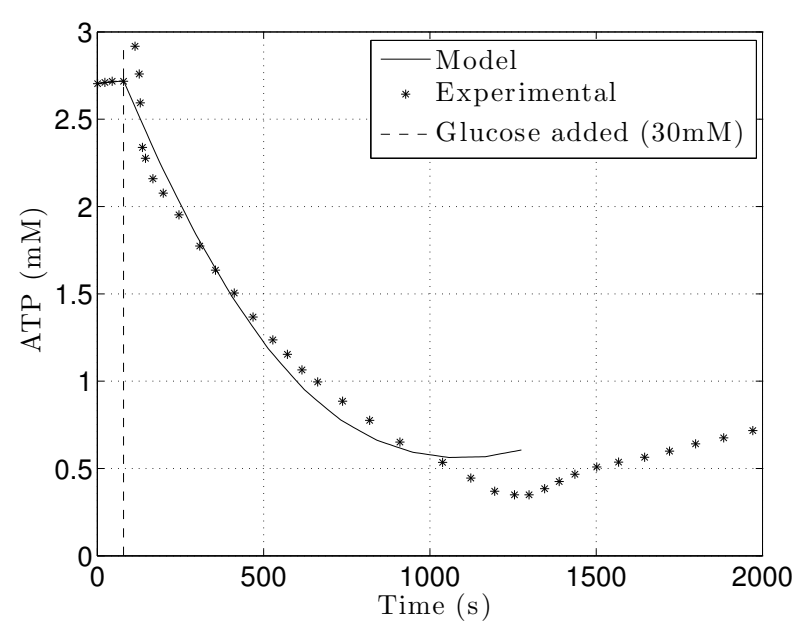

(a) Prediction of expected ATP level over time (in $\mathrm{mM}$ ) and experimental time-series.

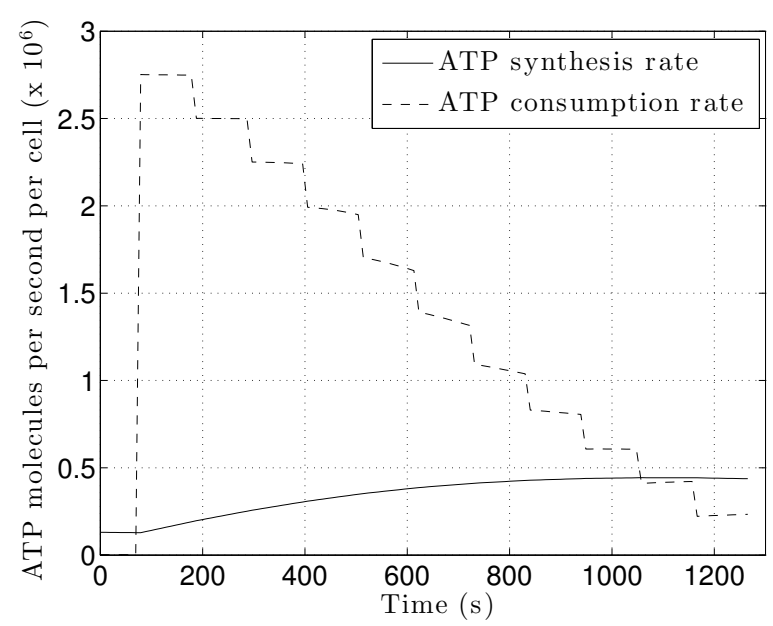

(c) Prediction of expected ATP synthesis and consumption rates per cell.

Figure 5.

determined after $t \simeq 1300 \mathrm{~s}$, rendering the corresponding experimental data useless.

With this approach, the estimated parameters, used in (31) to compute the corresponding flow rates, are given by

$$
\begin{cases}\hat{\gamma}=0 & \text { units } / \mathrm{mM} / \mathrm{s} \\ \hat{\rho}=2.31 \times 10^{-3} & \text { units } / \mathrm{mM} / \mathrm{s} \\ \hat{\zeta}=4.866 \times 10^{-3} & \text { units } / \mathrm{s} \\ \hat{\beta}=0.850 \times 10^{-3} & \text { units } / \mathrm{mM} / \mathrm{s}\end{cases}
$$

where "units", equivalent to $0.864 \times 10^{8}$ electrons, refers to the number of slots being occupied/emptied in the respective queue of the stochastic model, and $\mathrm{mM}$ refers to the glucose ED concentration. In particular, the "units" can be converted back into the original scale (36), which is related to the overall cell culture. The corresponding quantity related to a single cell is then obtained by converting one "unit" to the corresponding molecular quantity ( 1 unit=1.08 $\times$ $10^{8}$ ATP molecules $=0.432 \times 10^{8} \mathrm{NADH}$ molecules).

Figs. 5 a and 5 b plot, respectively, the ATP and NADH time-series, related to the cell culture, and the predicted

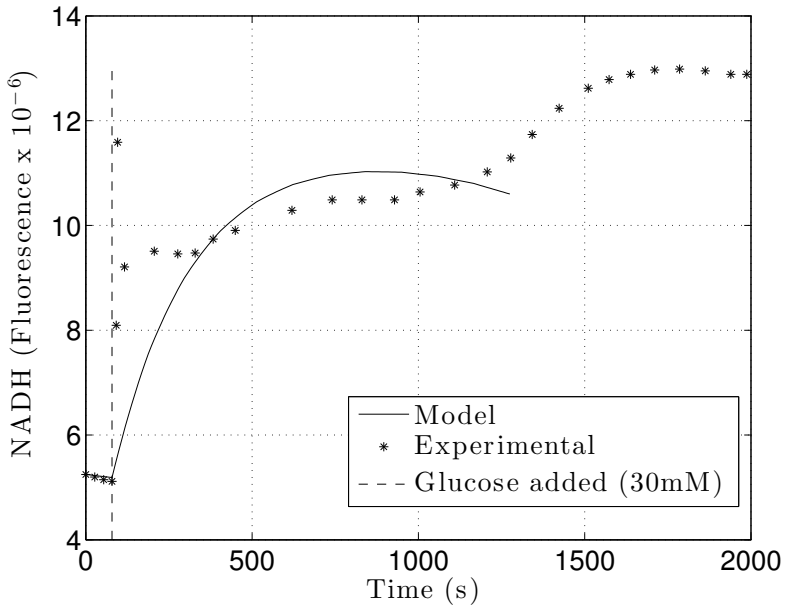

(b) Prediction of expected NADH level over time (equivalent fluorescence level) and experimental time-series.

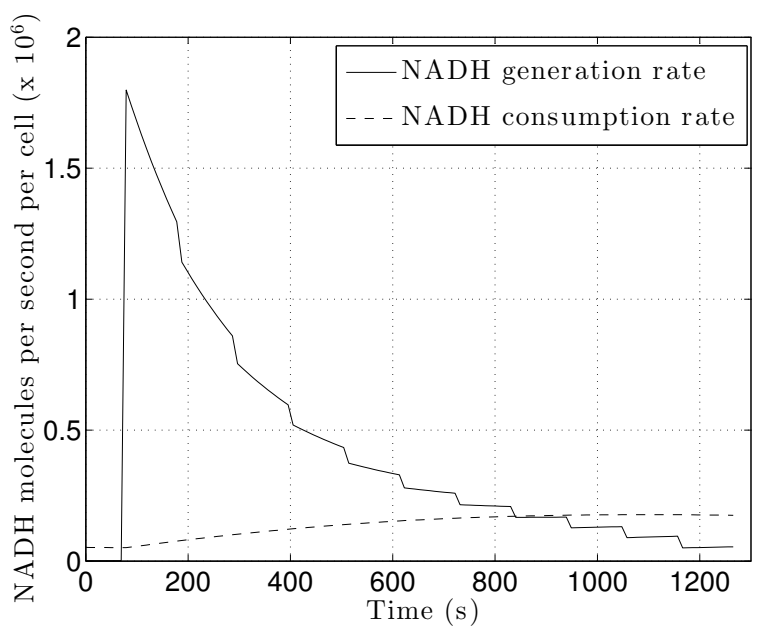

(d) Prediction of expected NADH generation and consumption rates per cell.

values based on our proposed stochastic model. We observe a good fit in the time-interval $t \in[0 \mathrm{~s}, 1300 \mathrm{~s}]$. The corresponding standard deviation of the error between the prediction and experimental curves is $0.1325[\mathrm{mM}]$ and 1.7736[Fluorescence $\times 10^{-6}$ ], respectively. The prediction error observed in the two figures can be explained by both cell lysis occurring in the bacterial population and the resulting distortion in the ATP and NADH levels, as explained in Remark 2, and by the bias introduced by our specific choice of the parametric model (31), which may not be sufficiently accurate to capture higher order fluctuations. The investigation of other parametric models to improve the prediction accuracy is left for future work. Figs. 5.c and 5.d plot, respectively, the expected ATP and NADH generation and consumption rates over time, related to a single cell. These biophysical parameters were not directly measured in the experiments by [34], but can be predicted by our proposed model. We notice that the ATP generation/consumption rate is of the order of $5 \times 10^{5} / 3 \times 10^{6}$, whereas the NADH generation/consumption rate is of the order of $2 \times 10^{6} / 2 \times 10^{5}$ (molecules per cell per second). These values are indeed physical, and consistent with 
known metabolic rates in yeast [39], which further motivates the development of this stochastic model as a predictive tool for microbial energetics.

\section{FUTURE WORK}

Our current experimental work involves measuring ATP and NADH levels in isolated single cells of bacterium $S$. oneidensis MR-1. This organism is capable of extracellular electron transfer by utilizing its array of outer-membrane multi-heme cytochromes and, due to its unique properties, presents a great model organism for this study. Similarly to the previous experiment done on the yeast Saccharomyces cerevisiae [34], whose data have been used in our experimental validation in Sec. $\mathrm{V}$, the ED will be abruptly added to a culture of starved bacterial cells, and, subsequently, the cellular ATP and NADH levels will be measured over time. As opposed to [34], we are developing an experimental setup in which the amount of the ED and the EA can be maintained at any desired level, therefore producing additional unprecedented data to be compared with our theoretical model.

The next step in our experiments will be to assemble bacterial cables, e.g., using similar techniques as in [40], and perform ATP and NADH measurements in these cells as the availability and the type of the ED and the EA is varied. In order to control and stimulate the growth of bacterial cables, a population of cells will be initially grown in an ED/EA rich medium, and subsequently moved to an environment with limited amounts of ED/EA, causing cell growth to stop. Then, the cells will be placed in a microfluidic medium where they can be moved, e.g., via optical tweezers [33], to form a one-dimensional bacterial cable. Adjusting the environmental parameters will then induce the production of electron transfer components in the bacteria, thus enabling long-range electron transfer in the cable. By keeping the concentration of ED/EA along the cable small, the bacteria are forced to use the externally provided solid-state electrodes as the electron source/sink and maintain the collective electron transfer through the cable. The role of each cell in this collective behavior is in the form of establishing direct cell-cell contact and facilitating electron transfer to and from the adjacent cells, and cooperation of every single cell in the system is necessary to provide enough ED/EA to sustain the entire network. Solid-phase electrodes poised to a desired electric potential can be used as the EA for such a cable. The rate of electron transfer to such an electrode can be controlled by adjusting its potential, and this electron transfer rate to the electrode can be accurately measured. Similar manipulations of the ED and the EA and their availability and the subsequent measurements on the state of the cells and the transfer rates within the chain will result in a vast amount of quantitative data to validate our stochastic model for IET.

\section{CONCLUSIONS}

In this paper, we have presented a stochastic model for electron transfer in bacterial cables. In particular, we have specialized the stochastic model to the case of an isolated cell, which is the building block of more complex bacterial cables, and we have provided an example of the application to the computation of the cell's lifetime. Moreover, we have designed a parameter estimation framework, based on a parametric description of the model, guided by biological constraints. The parameters were fit to experimental data available in the literature, demonstrating the capability of the proposed stochastic model to predict salient features related to the energetic state of the cells, such as ATP generation and consumption rates. This study is a first step towards addressing questions of more communications theoretic relevance, such as the interplay between information capacity of a microbial community and lifetime of the cells, reliability and delay in electron-based nanonetworks.

\section{REFERENCES}

[1] N. Michelusi, S. Pirbadian, M. Y. El-Naggar, and U. Mitra, "A model for electron transfer and cell energetics in bacterial cables," in 48th Annual Conference on Information Sciences and Systems (CISS), March 2014, pp. $1-6$.

[2] M. B. Miller and B. L. Bassler, "Quorum sensing in bacteria," Annual Review of Microbiology, vol. 55, no. 1, pp. 165-199, 2001.

[3] K. L. Visick and C. Fuqua, "Decoding Microbial Chatter: CellCell Communication in Bacteria," Journal of Bacteriology, vol. 187, no. 16, pp. 5507-5519, 2005. [Online]. Available: http: //jb.asm.org/content/187/16/5507.short

[4] K. H. K. H. Nealson, T. Platt, and J. W. Hastings, "Cellular Control of the Synthesis and Activity of the Bacterial Luminescent System," J. Bacteriol., vol. 104, no. 1, pp. 313-322, Oct. 1970.

[5] IEEE P1906.1 - Recommended Practice for Nanoscale and Molecular Communication Framework. [Online]. Available: https://standards.ieee. org/develop/project/1906.1.html

[6] S. F. Bush, Nanoscale Communication Networks. Artech House, 2010.

[7] T. Nakano, A. W. Eckford, and T. Haraguchi, Molecular Communication. Cambridge University Press, 2013.

[8] I. F. Akyildiz, F. Brunetti, and C. Blázquez, "Nanonetworks: A new communication paradigm," Computer Networks, vol. 52, no. 12, pp. 2260-2279, Aug. 2008. [Online]. Available: http: //dx.doi.org/10.1016/j.comnet.2008.04.001

[9] I. S. Mian and C. Rose, "Communication theory and multicellular biology," Integr. Biol., vol. 3, pp. 350-367, 2011.

[10] A. Eckford, "Nanoscale Communication with Brownian Motion," in 41st Annual Conference on Information Sciences and Systems (CISS), 2007, pp. 160-165.

[11] S. Kadloor, R. Adve, and A. Eckford, "Molecular Communication Using Brownian Motion With Drift," IEEE Transactions on NanoBioscience, vol. 11, no. 2, pp. 89-99, 2012.

[12] A. Einolghozati, M. Sardari, A. Beirami, and F. Fekri, "Capacity of discrete molecular diffusion channels," in IEEE International Symposium on Information Theory Proceedings (ISIT), 2011, pp. 723-727.

[13] — " "Data gathering in networks of bacteria colonies: Collective sensing and relaying using molecular communication," in INFOCOM Workshops, 2012, pp. 256-261.

[14] H. Arjmandi, A. Gohari, M. Kenari, and F. Bateni, "Diffusion-Based Nanonetworking: A New Modulation Technique and Performance Analysis," IEEE Communications Letters, vol. 17, no. 4, pp. 645-648, 2013.

[15] R. Mosayebi, H. Arjmandi, A. Gohari, M. N. Kenari, and U. Mitra, "On bounded memory decoder for molecular communications," in Information Theory and Applications Workshop (ITA), Feb. 2014.

[16] M. Moore, T. Suda, and K. Oiwa, "Molecular Communication: Modeling Noise Effects on Information Rate," IEEE Transactions on NanoBioscience, vol. 8, no. 2, pp. 169-180, 2009.

[17] M. Ş. Kuran, H. B. Yilmaz, T. Tugcu, and B. Özerman, "Energy model for communication via diffusion in nanonetworks," Nano Communication Networks, vol. 1, no. 2, pp. 86-95, 2010.

[18] G. Reguera, "When microbial conversations get physical," Trends in Microbiology, vol. 19(3), pp. 105-113, 2011.

[19] C. Pfeffer et al., "Filamentous bacteria transport electrons over centimetre distances," Nature, vol. 491(7423), pp. 218-221, 2012.

[20] S. Kato, K. Hashimoto, and K. Watanabe, "Microbial interspecies electron transfer via electric currents through conductive minerals," Proceedings of the National Academy of Sciences, vol. 109, no. 25, pp. 10042-10046, June 2012. 
[21] D. Gunduz, K. Stamatiou, N. Michelusi, and M. Zorzi, "Designing intelligent energy harvesting communication systems," IEEE Communications Magazine, vol. 52, no. 1, pp. 210-216, Jan. 2014.

[22] N. Michelusi, K. Stamatiou, and M. Zorzi, "Transmission Policies for Energy Harvesting Sensors with Time-Correlated Energy Supply," IEEE Transactions on Communications, vol. 61, no. 7, pp. 2988-3001, 2013.

[23] L. Liu, R. Zhang, and K.-C. Chua, "Wireless Information Transfer with Opportunistic Energy Harvesting," IEEE Transactions on Wireless Communications, vol. 12, no. 1, pp. 288-300, 2013.

[24] M. Y. El-Naggar and S. E. Finkel, "Live Wires: Electrical Signaling Between Bacteria," The Scientist, vol. 27, no. 5, pp. 38-43, 2013.

[25] M. Y. El-Naggar, G. Wanger, K. M. Leung, T. D. Yuzvinsky, G. Southam, J. Yang, W. M. Lau, K. H. Nealson, and Y. A. Gorby, "Electrical transport along bacterial nanowires from Shewanella oneidensis MR-1," Proceedings of the National Academy of Sciences, vol. 107(42), pp. 18 127-18 131, 2010.

[26] S. Pirbadian and M. Y. El-Naggar, "Multistep hopping and extracellular charge transfer in microbial redox chains," Physical Chemistry Chemical Physics, vol. 14, pp. 13 802-13808, 2012.

[27] G. Reguera, K. D. McCarthy, T. Mehta, J. S. Nicoll, M. T. Tuominen, and D. R. Lovley, "Extracellular electron transfer via microbial nanowires," Nature, vol. 435(7045), pp. 1098-1101, 2005.

[28] A. Rustom, R. Saffrich, I. Markovic, P. Walther, and H.-H. Gerdes, "Nanotubular Highways for Intercellular Organelle Transport," Science, pp. 1007-1010, Feb. 2004.

[29] J. P. Remis, D. Wei, A. Gorur, M. Zemla, J. Haraga, S. Allen, H. E. Witkowska, J. W. Costerton, J. E. Berleman, and M. Auer, "Bacterial social networks: structure and composition of Myxococcus xanthus outer membrane vesicle chains," Environmental Microbiology, vol. 16, no. 2, pp. 598-610, 2014. [Online]. Available: http: //dx.doi.org/10.1111/1462-2920.12187

[30] M. Kuran, H. Yilmaz, T. Tugcu, and I. Akyildiz, "Modulation Techniques for Communication via Diffusion in Nanonetworks," in IEEE International Conference on Communications (ICC), June 2011, pp. 15 .

[31] N. Lane, "Why Are Cells Powered by Proton Gradients?" Nature Education, vol. 3(9):18, 2010.

[32] W. Smith and J. Hashemi, Foundations of Materials Science and Engineering, ser. McGraw-Hill series in materials science and engineering. McGraw-Hill, 2003.

[33] H. Liu, G. Newton, R. Nakamura, K. Hashimoto, and S. Nakanishi, "Electrochemical Characterization of a Single Electricity-Producing Bacterial Cell of Shewanella by Using Optical Tweezers," Angewandte Chemie International Edition, vol. 49, pp. 6596-6599, Sep. 2010.

[34] V. Ozalp, P. T.R., N. L.J., and O. L.F., "Time-resolved measurements of intracellular ATP in the yeast Saccharomyces cerevisiae using a new type of nanobiosensor," Journal of Biological Chemistry, pp. 37579 37588 , Nov. 2010.

[35] G. H. Golub and C. F. Van Loan, Matrix computations (3rd ed.). Baltimore, MD, USA: Johns Hopkins University Press, 1996

[36] S. Boyd and L. Vandenberghe, Convex Optimization. New York, NY, USA: Cambridge University Press, 2004.

[37] P. Boggs, P. Domich, and J. Rogers, "An interior point method for general large-scale quadratic programming problems," Annals of Operations Research, vol. 62, no. 1, pp. 419-437, 1996.

[38] J. M. Berg, J. L. Tymoczko, and L. Stryer, Biochemistry, 5th ed. W H Freeman, 2002

[39] B. Teusink, J. Diderich, H. Westerhoff, K. Van Dam, and M. Walsh, "Intracellular glucose concentration in derepressed yeast cells consuming glucose is high enough to reduce the glucose transport rate by $50 \%$," Journal of Bacteriology, vol. 180(3), pp. 556-562, Feb. 1998

[40] P. Wang, L. Robert, J. Pelletier, W. Dang, F. Taddei, A. Wright, and S. Junemail, "Robust Growth of Escherichia coli," Current Biology, vol. 20, pp. 1099-1103, June 2010.

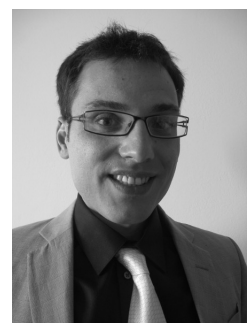

Nicolò Michelusi (S'09, M'13) received the B.Sc. (with honors), M.Sc. (with honors) and Ph.D. degrees from the University of Padova, Italy, in 2006, 2009 and 2013, respectively, and the M.Sc. degree in Telecommunications Engineering from the Technical University of Denmark in 2009, as part of the T.I.M.E. double degree program. In 2011, he was at the University of Southern California, Los Angeles, USA, and, in Fall 2012, at Aalborg University, Denmark, as a visiting research scholar. He is currently a post-doctoral research fellow at the Ming Hsieh Department of Electrical Engineering, University of Southern California, USA. His research interests lie in the areas of wireless networks, stochastic optimization, distributed estimation and modeling of bacterial networks. Dr. Michelusi serves as a reviewer for the IEEE Transactions on Communications, IEEE Transactions on Wireless Communications, IEEE Transactions on Information Theory, IEEE Transactions on Signal Processing, IEEE Journal on Selected Areas in Communications, and IEEE/ACM Transactions on Networking.

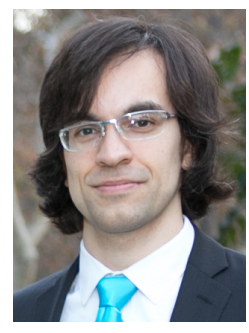

Sahand Pirbadian is a Ph.D. candidate in the Department of Physics and Astronomy at the University of Southern California. He received his B.Sc. degree in physics from Sharif University of Technology, Iran in 2010. His research interests include extracellular electron transfer (EET), electron transport in bacterial communities, bacterial multiheme cytochromes and bacterial nanowires. 


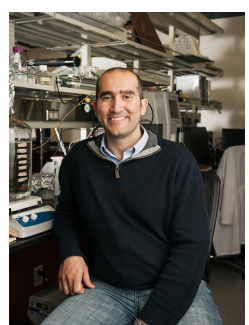

Moh El-Naggar is an assistant professor of physics at the University of Southern California's Dornsife College of Letters, Arts and Sciences. El-Naggar studies energy conversion and charge transmission at the interface between living cells and natural or synthetic surfaces. In 2012, he was named one of Popular Sciences Brilliant 10, and in 2014 he was awarded the Presidential Early Career Award for Scientists and Engineers (PECASE). El-Naggars work has important implications for cell physiology and astrobiology: it may lead to the development of new hybrid materials and renewable energy technologies that combine the exquisite biochemical control of nature with the synthetic building blocks of nanotechnology. El-Naggar earned his B.S. degree in mechanical engineering from Lehigh University (2001), and his Ph.D. in engineering and applied science from the California Institute of Technology (2007).

Urbashi Mitra received the B.S. and the M.S. degrees from the University of California at Berkeley and her Ph.D. from Princeton University. After a six year stint at the Ohio State University, she joined the Department of Electrical Engineering at the University of Southern California, Los Angeles, where she is currently a Professor. She is a member of the IEEE Information Theory Society's Board of Governors (2002-2007, 2012-2014) and the IEEE Signal Processing Societys Technical Committee on Signal Processing for Communications and Networks (2012-2014). Dr. Mitra is a Fellow of the IEEE. She is the recipient of: 2012 Globecom Signal Processing for Communications Symposium Best Paper Award, 2012 NAE Lillian Gilbreth Lectureship, USC Center for Excellence in Research Fellowship (2010-2013), the 2009 DCOSS Applications \& Systems Best Paper Award, Texas Instruments Visiting Professor (Fall 2002, Rice University), 2001 Okawa Foundation Award, 2000 OSU College of Engineering Lumley Award for Research, 1997 OSU College of Engineering MacQuigg Award for Teaching, and a 1996 National Science Foundation (NSF) CAREER Award. Dr. Mitra currently serves on the IEEE Fourier Award for Signal Processing committee and the IEEE James H. Mulligan, Jr. Education Medal committee. She has been/is an Associate Editor for the following IEEE publications: Transactions on Signal Processing (2012-), Transactions on Information Theory (2007-2011), Journal of Oceanic Engineering (2006-2011), and Transactions on Communications (1996-2001). She has co-chaired: (technical program) 2014 IEEE International Symposium on Information Theory in Honolulu, HI, 2014 IEEE Information Theory Workshop in Hobart, Tasmania, IEEE 2012 International Conference on Signal Processing and Communications, Bangalore India, and the IEEE Communication Theory Symposium at ICC 2003 in Anchorage, AK; and general co-chair for the first ACM Workshop on Underwater Networks at Mobicom 2006, Los Angeles, CA Dr. Mitra was the Tutorials Chair for IEEE ISIT 2007 in Nice, France and the Finance Chair for IEEE ICASSP 2008 in Las Vegas, NV. Dr. Mitra has held visiting appointments at: the Delft University of Technology, Stanford University, Rice University, and the Eurecom Institute. She served as co-Director of the Communication Sciences Institute at the University of Southern California from 2004-2007. Her research interests are in: wireless communications, communication and sensor networks, detection and estimation and the interface of communication, sensing and control. 